\title{
Residual-based stabilization of the finite element approximation to the acoustic perturbation equations for low Mach number aeroacoustics
}

\author{
Oriol Guasch ${ }^{1 *}$, Patrícia Sánchez-Martín ${ }^{1}$, Arnau Pont $^{2}$, Joan Baiges ${ }^{2}$, Ramon \\ Codina $^{2}$ \\ ${ }^{1}$ GTM - Grup de recerca en Tecnologies Mèdia, La Salle, Universitat Ramon Llull, C/ Quatre Camins \\ 2, 08022 Barcelona, Catalonia \\ ${ }^{2}$ Universitat Politècnica de Catalunya, C/ Jordi Girona 1-3, Edifici C1, 08034 Barcelona, Catalonia
}

SUMMARY

The acoustic perturbation equations (APE) are suitable to predict aerodynamic noise in the presence of a non-uniform mean flow. As for any hybrid computational aeroacoustics approach, a first computational fluid dynamics simulation is carried out from which the mean flow characteristics and acoustic sources are obtained. In a second step, the APE are solved to get the acoustic pressure and particle velocity fields. However, resorting to the finite element method (FEM) for that purpose is not straightforward. Whereas mixed finite elements satisfying an appropriate inf-sup compatibility condition can be built in the case of no mean flow, i.e., for the standard wave equation in mixed form, these are difficult to implement and their good performance is yet to be checked for more complex wave operators. As a consequence, strong simplifying assumptions are usually considered when solving the APE with FEM. It is possible to avoid them by resorting to stabilized formulations. In this work, a residual-based stabilized FEM is presented for the APE at low Mach numbers, which allows one to deal with the APE convective and reaction terms in its full extent. The key of the approach resides in the design of the matrix of stabilization parameters. The performance of the formulation and the contributions of the different terms in the equations are tested for an acoustic pulse propagating in sheared solenoidal mean flow, and for the aeolian tone generated by flow past a two-dimensional cylinder. Copyright (C) 2015 John Wiley \& Sons, Ltd.

Received ...

KEY WORDS: Acoustics; Aeroacoustics; Stabilized FEM; VMS: Variational Multiscale; Finite element; Subsonic

\section{INTRODUCTION}

In this work, a stabilized finite element method (FEM) is proposed to solve the acoustic perturbation equations (APE). These equations can account for aerodynamic sound propagation in areas with non-uniform mean flow. In particular, we will herein focus on the low Mach number APE in [1], which are derived after source filtering the linearized Euler equations (LEE) to get rid of the vorticity and entropy modes, while leaving the acoustic ones.

The APE constitute a particular case of the so called hybrid methods in computational aeroacoustics (CAA). These separate the computation of aerodynamic sound into two steps.

*Correspondence to: ${ }^{1}$ oguasch@salleurl.edu,www.salle.url.edu/ oguasch

Copyright (c) 2015 John Wiley \& Sons, Ltd.

Prepared using fldauth.cls [Version: 2010/05/13 v2.00] 
In the first one, a computational fluid dynamics (CFD) simulation is carried out to obtain the acoustic source terms from the aerodynamic velocity and pressure fields. In the second one, the source terms are input into an acoustic wave operator. The latter can directly be, for example, the standard linear wave equation in irreducible or mixed form, their convective counterparts, or more complex operators like the ones involved in the LEE.

The best known hybrid approaches are those in the category of acoustic analogies, being Lighthill's the most celebrated one [2]. Lighthill's analogy states the problem of aerodynamic sound radiation into a quiescent medium as that of noise radiated by a distribution of quadrupoles in free space. For low Mach number flows, Lighthill approximates the source of sound by the double divergence of the Reynolds tensor, which is built from the products of the components of the aerodynamic velocity vector. Other analogies place their emphasis on the role of vorticity in the generation of sound $[3,4,5]$. Besides, it should be remarked that the influence of rigid bodies within the flow on noise radiation was considered since the birth of acoustic analogies (see [6]) and later generalized to flexible bodies in [7].

As long as one aims to compute aerodynamic sound propagation in non-quiescent flow areas, convective and refraction effects have to be extracted from the acoustic source term and included in the wave operator (see e.g., [8]). This led to the development of more sophisticated analogies (see e.g., $[9,10]$ ) and to alternatives such as the linearized Euler equations (LEE) [11], or resorting to perturbation equations [1, 12]. As mentioned above, in this work we will attempt at solving a low Mach number formulation of the APE in [1] using FEM. Refraction effects will be neglected for simplicity.

The computation of aerodynamic sound in the second step of a hybrid CAA approach is often performed by resorting to integral formulations, though FEM is also common if the acoustic waves are to be computed at distances not too far from the source region. Most aeroacoustic FEM works to date have dealt with Lighthill's acoustic analogy, [13, 14, 15, 16], yet convection effects have also been taken into account. In [17] the convective Helmholtz equation flow was considered, while a mixed convection wave equation with uniform mean flow was considered in [18] as a simplification of the second variant of the APE in [1].

The numerical difficulties associated to the FEM solution of the various wave operators involved in CAA are of a very different nature. Whereas for the standard linear wave equation for the acoustic pressure most efforts are placed on the time discretization schemes, given that the spatial discretization presents no serious difficulties (the Laplacian is a wellbehaved operator), this is not the case for its Fourier transform, the Helmholtz equation. The latter may become non-positive definite for large wavenumbers and the standard Galerkin FEM approach fails to solve it. A large amount of stabilization strategies have been devised to prevent the pollution error associated to the Helmholtz equation [19]. With regard to mixed formulations like the APE, which involve both, the acoustic pressure and the acoustic particle velocity, the situation becomes more intricate. In the case of no convection the APE simply reduces to the wave equation in mixed form. The variational formulation of the latter has to satisfy a compatibility inf-sup condition for the problem to be well posed, which is not inherited by the standard Galerkin FEM approach to it. It therefore becomes necessary to work with finite elements having different interpolations for the acoustic pressure and velocity fields to avoid the appearance of spurious oscillations in the numerical solution [20,21, 22]. An alternative to circumvent the discrete inf-sup condition and consider the same interpolation fields for the acoustic pressure and velocity is that of resorting to stabilization strategies, such as the residual based multiscale methods [23, 24, 25]. These were applied to the mixed wave equation in [26]. It was proved in [27] that the solution to the problem is then bounded by the data in an energy norm that involves all additional stabilization terms.

The situation worsens when one considers the presence of a mean flow. As far as the authors know, it has not been checked yet whether the tailored finite elements for the mixed wave equation could perform well in the presence of convection. Even if this was the case, their implementation is not an easy task. Stabilized FEM offer a way out to these problems. 
A first simplification to the APE is that of assuming a uniform mean flow so that they reduce to the convective mixed wave equation. As previously commented, this occurrence was addressed in [18], where a stabilizing flux term (as in the discontinuous Galerkin method) and a penalization term were incorporated in the formulation. However, in the case of a non-uniform mean flow, not only the convective terms in the APE become more complex, but one also has to deal with a reaction term. With regard to the former, analogous nonuniform convective terms appear in the linearized modified Boussinesq equation for shallow waters. The Galerkin FEM approach to it exhibits high frequency oscillations [28, 29] that can be overcome by resorting to stabilization strategies [30, 31]. The same type of instabilities are found when setting the wave equation in mixed form in an arbitrary Lagrangian-Eulerian (ALE) frame of reference, to deal with wave propagation in moving domains [32]. Stabilization strategies have been also applied to the LEE. In particular, the discontinuous Galerkin and the stream upwind Petrov-Galerkin (SUPG) approaches were tested in [33] to suppress the artificial growth of the Kelvin-Helmholtz instabilities in the LEE (these instabilities are limited in real flows by non-linear effects). Furthermore, the algebraic subgrid scale (ASGS) method in [17] was used to stabilize the standard Galerkin FEM approach to the LEE in [34].

The contributions of this work are as follows. First, a residual-based stabilized FEM is developed to solve a simplified version of the second variant of the APE equations (see $[1,18]$ ), that includes the effects of non-uniform convection and of the reaction term due to the mean velocity gradient. As far as the authors know neither these effects had been taken into account in previous FEM approaches to the APE, nor residual-based FEM methods have been applied to them. Besides, the implemented stabilization strategy relies on splitting the problem unknowns into large scales that can be resolved by the computational mesh, and small scales whose effects onto the large scales have to be modelled. Its main advantage is that equal degree of interpolation can be used for the acoustic pressure and particle velocity fields. Yet, the key for the good performance of the method depends upon the design of a proper matrix of stabilization parameters, which constitutes the second main goal of the work. This is achieved through a Fourier analysis of the subscale equation following the lines in [26, 31, 32]. Finally, the importance of the various terms in the analyzed APE system is highlighted by means of some numerical examples. The latter reveal that neglecting some of the terms to simplify the equations may result in significant deviations in the wavefronts of the predicted aerodynamic sound.

The paper is organized as follows. In section 2, we disclose the APE equations, set them in matrix form and work out their variational formulation. In section 3, we proceed to discretization. The residual-based stabilized FEM approach is presented and the derivation of the matrix of stabilization parameters is exposed in detail. The fully discretized numerical scheme in time and space close the section. Two numerical examples are finally given in section 4 . These consist of two benchmark tests dealing with the wavefront propagation in a solenoidal sheared mean flow and with the generation of an aeolian tone by flow past a two-dimensional cylinder.

\section{PROBLEM STATEMENT}

\subsection{An acoustic perturbation equation for low Mach numbers}

Our starting point will be the acoustic perturbation equations (53)-(55) derived in [1], in the particular case of only considering vortex sound for low Mach numbers and neglecting 
the linear coupling between acoustic and vorticity modes. These equations read

$$
\begin{aligned}
\partial_{t} \rho^{\prime}+\nabla \cdot\left(\rho^{\prime} \overline{\boldsymbol{u}}+\bar{\rho} \boldsymbol{u}^{a}\right) & =0, \\
\partial_{t} \boldsymbol{u}^{a}+\nabla\left(\overline{\boldsymbol{u}} \cdot \boldsymbol{u}^{a}\right)+\nabla\left(\frac{p^{a}}{\bar{\rho}}\right) & =\mathbf{0}, \\
\partial_{t} p^{a}-\bar{c}^{2} \partial_{t} \rho^{\prime} & =-\partial_{t} p^{h},
\end{aligned}
$$

and are obtained from manipulation of the linearized Euler equations for the enthalpy and velocity fields (the reader is referred to [1] for details). $\partial_{t}$ stands for the first order time derivative and $\bar{c}$ denotes the speed of sound. The above equations are based on the following decompositions for the compressible velocity $\boldsymbol{u}$, pressure $p$ and density $\rho$,

$$
\begin{array}{rll}
\boldsymbol{u}=\overline{\boldsymbol{u}}+\boldsymbol{u}^{\prime} & \text { with } & \boldsymbol{u}^{\prime}:=\boldsymbol{u}^{h}+\boldsymbol{u}^{a}, \\
p=\bar{p}+p^{\prime} & \text { with } & p^{\prime}:=p^{h}+p^{a}, \\
\rho=\bar{\rho}+\rho^{\prime} & \text { with } & \rho^{\prime}:=\rho^{h}+\rho^{a} .
\end{array}
$$

In (1)-(2) an overbar indicates a time averaged mean quantity i.e., $\overline{g(\boldsymbol{x})}:=$ $\lim _{T \rightarrow \infty}(1 / T) \int_{t_{0}}^{t_{0}+T} g(\boldsymbol{x}, t) d t$, with $t_{0}$ standing for the initial time of the averaging process and $T$ for its total duration. The mean values are independent of $t_{0}$ once the initial transients of a simulation/measurement have been surpassed. Likewise, a prime $g(\boldsymbol{x}, t)^{\prime}$ denotes a perturbation from the mean value. The velocity fluctuation $\boldsymbol{u}^{\prime}$ in (2a) becomes split into two components, a hydrodynamic solenoidal perturbation $\boldsymbol{u}^{h}$ and an irrotational acoustic perturbation $\boldsymbol{u}^{a}$. Similarly, the pressure and density fluctuations $p^{\prime}$ and $\rho^{\prime}$ in (2b)-(2c) consist of two terms, a hydrodynamic component $p^{h}$ and $\rho^{h}$ responsible for the production of pseudo-sound, and an acoustic component $p^{a}$ and $\rho^{a}$ that will propagate as acoustic waves outwards the source region.

Equation (1) can be further simplified assuming that neither the time averaged sound speed nor the time averaged density have a spatial dependence, i.e., $\bar{c} \equiv c_{0}$ and $\bar{\rho} \equiv \rho_{0}$. Moreover and given that we are dealing with low Mach flows, we can resort to an incompressible computational fluid dynamics (CFD) simulation to compute the mean hydrodynamic velocity $\overline{\boldsymbol{u}}$ and the hydrodynamic pressure $p^{h}$. In such situation we get $\nabla \cdot \overline{\boldsymbol{u}}=0$ and assuming $p^{\prime}=c_{0}^{2} \rho^{\prime}$, equation (1) simplifies to

$$
\begin{gathered}
\frac{1}{\rho_{0} c_{0}^{2}} \partial_{t} p^{a}+\frac{1}{\rho_{0} c_{0}^{2}} \overline{\boldsymbol{u}} \cdot \nabla p^{a}+\nabla \cdot \boldsymbol{u}^{a}=-\frac{1}{\rho_{0} c_{0}^{2}} \partial_{t} p^{h}-\frac{1}{\rho_{0} c_{0}^{2}} \overline{\boldsymbol{u}} \cdot \nabla p^{h}, \\
\rho_{0} \partial_{t} \boldsymbol{u}^{a}+\rho_{0} \overline{\boldsymbol{u}} \cdot \nabla \boldsymbol{u}^{a}+\nabla p^{a}+\rho_{0} \boldsymbol{u}^{a} \cdot \nabla \overline{\boldsymbol{u}}=\mathbf{0} .
\end{gathered}
$$

Equation (3) constitutes the simplification proposed in [18] for the APE variant 2 in [1]. It only involves the acoustic pressure and acoustic particle velocity as the problem unknowns. In the present work we will extend the analysis in [18] by considering non uniform mean velocity flows. Notice that the reaction term $\rho_{0} \boldsymbol{u}^{a} \cdot \nabla \overline{\boldsymbol{u}}$ does not vanish in such cases.

\subsection{Differential matrix problem and variational formulation}

It is possible to rewrite equations (3a) and (3b) in matrix form for convenience as

$$
\boldsymbol{\mu} \partial_{t} \boldsymbol{U}+\boldsymbol{A}_{i} \partial_{i} \boldsymbol{U}+\boldsymbol{S} \boldsymbol{U}=\boldsymbol{F}
$$

where the summation convention over repeated indexes is assumed hereinafter. The index $i$ will run from 1 to the number of spatial dimensions with $\partial_{i}$ standing for the first order 
spatial derivatives. The following vector and matrix identifications have been made

$$
\begin{gathered}
\boldsymbol{U}=\left(\begin{array}{l}
p^{a} \\
u_{1}^{a} \\
u_{2}^{a} \\
u_{3}^{a}
\end{array}\right), \boldsymbol{F}=\left(\begin{array}{l}
Q \\
f_{1} \\
f_{2} \\
f_{3}
\end{array}\right), \boldsymbol{\mu}=\left(\begin{array}{cccc}
\mu_{p} & 0 & 0 & 0 \\
0 & \mu_{\boldsymbol{u}} & 0 & 0 \\
0 & 0 & \mu_{\boldsymbol{u}} & 0 \\
0 & 0 & 0 & \mu_{\boldsymbol{u}}
\end{array}\right), \boldsymbol{A}_{1}=\left(\begin{array}{cccc}
\mu_{p} \bar{u}_{1} & 1 & 0 & 0 \\
1 & \mu_{\boldsymbol{u}} \bar{u}_{1} & 0 & 0 \\
0 & 0 & \mu_{\boldsymbol{u}} \bar{u}_{1} & 0 \\
0 & 0 & 0 & \mu_{\boldsymbol{u}} \bar{u}_{1}
\end{array}\right) \\
\boldsymbol{A}_{2}=\left(\begin{array}{cccc}
\mu_{p} \bar{u}_{2} & 0 & 1 & 0 \\
0 & \mu_{\boldsymbol{u}} \bar{u}_{2} & 0 & 0 \\
1 & 0 & \mu_{\boldsymbol{u}} \bar{u}_{2} & 0 \\
0 & 0 & 0 & \mu_{\boldsymbol{u}} \bar{u}_{2}
\end{array}\right), \boldsymbol{A}_{3}=\left(\begin{array}{cccc}
\mu_{p} \bar{u}_{3} & 0 & 0 & 1 \\
0 & \mu_{\boldsymbol{u}} \bar{u}_{3} & 0 & 0 \\
0 & 0 & \mu_{\boldsymbol{u}} \bar{u}_{3} & 0 \\
1 & 0 & 0 & \mu_{\boldsymbol{u}} \bar{u}_{3}
\end{array}\right) \\
\boldsymbol{S}=\left(\begin{array}{cccc}
0 & 0 & 0 & 0 \\
0 & \mu_{\boldsymbol{u}} \partial_{1} \bar{u}_{1} & \mu_{\boldsymbol{u}} \partial_{2} \bar{u}_{1} & \mu_{\boldsymbol{u}} \partial_{3} \bar{u}_{1} \\
0 & \mu_{\boldsymbol{u}} \partial_{1} \bar{u}_{2} & \mu_{\boldsymbol{u}} \partial_{2} \bar{u}_{2} & \mu_{\boldsymbol{u}} \partial_{3} \bar{u}_{2} \\
0 & \mu_{\boldsymbol{u}} \partial_{1} \bar{u}_{3} & \mu_{\boldsymbol{u}} \partial_{2} \bar{u}_{3} & \mu_{\boldsymbol{u}} \partial_{3} \bar{u}_{3}
\end{array}\right) .
\end{gathered}
$$

The parameters $\mu_{p} \equiv\left(\rho_{0} c_{0}^{2}\right)^{-1}$ and $\mu_{\boldsymbol{u}} \equiv \rho_{0}$ satisfying $c_{0}=\left(\mu_{p} \mu_{\boldsymbol{u}}\right)^{-1 / 2}$ in (5) have been introduced to ease the notation and $\bar{u}_{i}, i=1,2,3$, designate the components of the time averaged mean velocity vector. Note also that, for completeness, we have included the possibility of an external force acting on the momentum equation by means of the components $f_{i}$.

Problem (4) is to be solved in a computational domain $\Omega$ whose boundary $\partial \Omega$ can be considered to be made from the union of two disjoint sets: $\Gamma_{p}$ where we will prescribe the acoustic pressure and $\Gamma_{\boldsymbol{u}}$ where the normal component of the acoustic particle velocity is to be imposed. For simplicity, we shall take homogeneous Dirichlet conditions on them in the forthcoming expressions, i.e., $p^{a}=0$ on $\Gamma_{p}, t>0$ and $\boldsymbol{u}^{a} \cdot \boldsymbol{n}=0$ on $\Gamma_{\boldsymbol{u}}, t>0$, with $\boldsymbol{n}$ standing for the outward normal of the domain's boundary. Equation (4) needs also to be suplemmented with initial conditions $p^{a}(\boldsymbol{x}, 0)=p_{0}^{a}(\boldsymbol{x})$ and $\boldsymbol{u}^{a}(\boldsymbol{x}, 0)=\boldsymbol{u}_{0}^{a}(\boldsymbol{x})$ in $\Omega$.

Additionally, let us introduce the functional spaces $V_{p}(\Omega)=\left\{q \in H^{1}(\Omega) \mid \overline{\boldsymbol{u}} \cdot \nabla q \in L^{2}(\Omega), q=\right.$ 0 on $\left.\Gamma_{p}\right\}$ and $\boldsymbol{V}_{\boldsymbol{u}}(\Omega)=\left\{\boldsymbol{v} \in \boldsymbol{L}^{2}(\Omega) \mid \nabla \cdot \boldsymbol{v} \in L^{2}(\Omega), \overline{\boldsymbol{u}} \cdot \nabla \boldsymbol{v} \in \boldsymbol{L}^{2}(\Omega), \boldsymbol{v} \cdot \boldsymbol{n}=0\right.$ on $\left.\Gamma_{\boldsymbol{u}}\right\}$ and use $(f, g)$ to denote the integral of the product of two arbitrary functions $f$ and $g$, i.e., $(f, g):=\int_{\Omega} f g d \Omega$. Analogously, for vector valued functions we will have $(\boldsymbol{f}, \boldsymbol{g}):=\int_{\Omega} \boldsymbol{f}^{\top} \boldsymbol{g} d \Omega$. Next, consider a test function $q$ for the acoustic pressure and $\boldsymbol{v}$ for the acoustic velocity. The weak form of (4) is found multiplying it by a vector test function $\boldsymbol{V} \equiv[q, \boldsymbol{v}]^{\top}$ and integrating over the computational domain $\Omega$. Defining the spaces $\mathcal{L}(\Omega) \equiv L^{2}(\Omega) \times \boldsymbol{L}^{2}(\Omega)$ and $\mathcal{V}(\Omega) \equiv V_{p}(\Omega) \times \boldsymbol{V}_{\boldsymbol{u}}(\Omega)$, the problem becomes that of finding $\boldsymbol{U} \equiv\left[p^{a}, \boldsymbol{u}^{a}\right]^{\top} \in C^{1}([0, T], \mathcal{L}(\Omega)) \cap C^{0}([0, T], \mathcal{V}(\Omega))$ such that

$$
\left(\boldsymbol{\mu} \partial_{t} \boldsymbol{U}, \boldsymbol{V}\right)+\left(\boldsymbol{A}_{i} \partial_{i} \boldsymbol{U}, \boldsymbol{V}\right)+(\boldsymbol{S U}, \boldsymbol{V})=(\boldsymbol{F}, \boldsymbol{V}) \quad \forall \boldsymbol{V} \in \mathcal{V}(\Omega) .
$$

\section{NUMERICAL APPROXIMATION}

\subsection{Residual-based stabilized finite element method}

The standard discretized conforming Galerkin FEM approach to solve problem (6) aims at finding a finite element solution $\boldsymbol{U}_{h} \in C^{1}\left([0, T], \mathcal{L}_{h}(\Omega) \subset \mathcal{L}(\Omega)\right) \cap C^{0}\left([0, T], \mathcal{V}_{h}(\Omega) \subset \mathcal{V}(\Omega)\right)$ such that

$$
\left(\boldsymbol{\mu} \partial_{t} \boldsymbol{U}_{h}, \boldsymbol{V}_{h}\right)+\left(\boldsymbol{A}_{i} \partial_{i} \boldsymbol{U}_{h}, \boldsymbol{V}_{h}\right)+\left(\boldsymbol{S} \boldsymbol{U}_{h}, \boldsymbol{V}_{h}\right)=\left(\boldsymbol{F}, \boldsymbol{V}_{h}\right) \quad \forall \boldsymbol{V}_{h} \in \mathcal{V}_{h}(\Omega) .
$$

Here, $\mathcal{L}_{h}(\Omega), \mathcal{V}_{h}(\Omega)$ represent finite dimensional spaces built from a finite element partition $\left\{\Omega_{e}\right\}$ of $\Omega$. The index $e$ ranges from 1 to the total number of elements $n_{e l}$ in the computational mesh. However, the Galerkin FEM formulation (7) is known to suffer from numerical instabilities which can be overcome by resorting to a variational multiscale stabilization approach. The latter basically consists in splitting the exact solution $\boldsymbol{U}$ into a large component $\boldsymbol{U}_{h}$ that can be captured by the finite element computational mesh, plus 
a small component $\boldsymbol{U}^{\prime}$, which cannot be resolved by the mesh. $\boldsymbol{U}^{\prime}$ is usually referred to as the subscale. The effects of the subscales onto the large scales have to be modeled. The procedure to do so give rise to different stabilization methods.

Substituting $\boldsymbol{U}=\boldsymbol{U}_{h}+\boldsymbol{U}^{\prime}$ into (6) results in two equations. The first one governs the dynamics of the large scales and is given by

$$
\left(\boldsymbol{\mu} \partial_{t} \boldsymbol{U}_{h}, \boldsymbol{V}_{h}\right)+\left(\boldsymbol{\mu} \partial_{t} \boldsymbol{U}^{\prime}, \boldsymbol{V}_{h}\right)+\left(\boldsymbol{A}_{i} \partial_{i} \boldsymbol{U}_{h}, \boldsymbol{V}_{h}\right)+\left(\boldsymbol{A}_{i} \partial_{i} \boldsymbol{U}^{\prime}, \boldsymbol{V}_{h}\right)+\left(\boldsymbol{S} \boldsymbol{U}_{h}, \boldsymbol{V}_{h}\right)+\left(\boldsymbol{S} \boldsymbol{U}^{\prime}, \boldsymbol{V}_{h}\right)=\left(\boldsymbol{F}, \boldsymbol{V}_{h}\right)
$$

To better appreciate the influence of the subscales in the large scale equation we can integrate the convective term by parts presuming the subscales to be local and vanish at the interelement boundaries. This is not a strong assumption given that no exact values can be found for the subscales; only their mean effects onto the large scales can be computed. Furthermore, we will assume the subscales to be quasi-static, i.e., $\partial_{t} \boldsymbol{U}^{\prime} \approx \mathbf{0}$. This hypothesis has proven valuable in previous stabilization works involving wave propagation phenomena, see e.g. $[17,26,31,14,32]$. On the other hand, the implementation of time evolving dynamic subscales has been considered, for example, in coupled problems involving the incompressible Navier-Stoles equations [35, 36, 37]. Yet for simplicity dynamic subscales will not be taken into account in this first work on stabilized FEM for the acoustic perturbation equations, that being left for future developments. All this leads to the following expression for equation (8),

$$
\begin{aligned}
& \left(\boldsymbol{\mu} \partial_{t} \boldsymbol{U}_{h}, \boldsymbol{V}_{h}\right)+\left(\boldsymbol{A}_{i} \partial_{i} \boldsymbol{U}_{h}, \boldsymbol{V}_{h}\right)+\left(\boldsymbol{S} \boldsymbol{U}_{h}, \boldsymbol{V}_{h}\right) \\
& -\left(\boldsymbol{U}^{\prime}, \boldsymbol{A}_{i}^{\top} \partial_{i} \boldsymbol{V}_{h}\right)-\left(\boldsymbol{U}^{\prime},\left[\partial_{i} \boldsymbol{A}_{i}^{\top}\right] \boldsymbol{V}_{h}\right)+\left(\boldsymbol{U}^{\prime}, \boldsymbol{S}^{\top} \boldsymbol{V}_{h}\right)=\left(\boldsymbol{F}, \boldsymbol{V}_{h}\right) .
\end{aligned}
$$

Notice that the first line in (9) contains the terms of the standard Galerkin approach in (7), whereas the second line discloses the subscale stabilization terms. The term $\left(\boldsymbol{U}^{\prime},\left[\partial_{i} \boldsymbol{A}_{i}^{\top}\right] \boldsymbol{V}_{h}\right)$ in the second line plays the role of an additional reaction weighted by the subscales; yet as seen from (5) this term automatically vanishes because we are considering solenoidal mean flows.

On the other hand, the second equation driving the dynamics of $\boldsymbol{U}^{\prime}$ has the expression

$$
\left(\boldsymbol{\mu} \partial_{t} \boldsymbol{U}_{h}, \boldsymbol{V}^{\prime}\right)+\left(\boldsymbol{A}_{i} \partial_{i} \boldsymbol{U}_{h}, \boldsymbol{V}^{\prime}\right)+\left(\boldsymbol{A}_{i} \partial_{i} \boldsymbol{U}^{\prime}, \boldsymbol{V}^{\prime}\right)+\left(\boldsymbol{S} \boldsymbol{U}_{h}, \boldsymbol{V}^{\prime}\right)+\left(\boldsymbol{S} \boldsymbol{U}^{\prime}, \boldsymbol{V}^{\prime}\right)=\left(\boldsymbol{F}, \boldsymbol{V}^{\prime}\right),
$$

which corresponds to the $L^{2}$-projection of $\boldsymbol{\mu} \partial_{t} \boldsymbol{U}_{h}+\boldsymbol{A}_{i} \partial_{i} \boldsymbol{U}_{h}+\boldsymbol{A}_{i} \partial_{i} \boldsymbol{U}^{\prime}+\boldsymbol{S} \boldsymbol{U}_{h}+\boldsymbol{S} \boldsymbol{U}^{\prime}=\boldsymbol{F}$ onto the space of subscales. If we use $\mathcal{P}$ to denote this projection, (10) can be rewritten as

$$
\mathcal{P}\left(\boldsymbol{A}_{i} \partial_{i} \boldsymbol{U}^{\prime}+\boldsymbol{S} \boldsymbol{U}^{\prime}\right)=\mathcal{P}\left[\boldsymbol{F}-\left(\boldsymbol{\mu} \partial_{t} \boldsymbol{U}_{h}+\boldsymbol{A}_{i} \partial_{i} \boldsymbol{U}_{h}+\boldsymbol{S} \boldsymbol{U}_{h}\right)\right]=: \boldsymbol{R}_{h},
$$

where the residual $\boldsymbol{R}_{h}$ of the finite element approximation onto the subscale space has been introduced. The goal of the residual-based stabilization approaches is to find an approximate solution to (11), which yields an expression for $\boldsymbol{U}^{\prime}$ that could be substituted in (9) resulting in enhanced stabilization properties.

A standard option consists in taking the subscales proportional to the residual i.e., $\boldsymbol{U}^{\prime}=$ $\boldsymbol{\tau} \boldsymbol{R}_{h}$, which in our case, is analogous to saying that $\mathcal{P}\left(\boldsymbol{A}_{i} \partial_{i} \boldsymbol{U}^{\prime}+\boldsymbol{S} \boldsymbol{U}^{\prime}\right) \approx \boldsymbol{\tau}^{-1} \boldsymbol{U}^{\prime}$, see $[26,31,32]$. Here, $\boldsymbol{\tau}$ stands for a symmetric, positive-definite matrix of stabilization parameters that has to be determined. Once this has been done, $\boldsymbol{U}^{\prime}=\boldsymbol{\tau} \boldsymbol{R}_{h}$ can be substituted into the large scale equation (9) that becomes

$$
\begin{aligned}
& \left(\boldsymbol{\mu} \partial_{t} \boldsymbol{U}_{h}, \boldsymbol{V}_{h}\right)+\left(\boldsymbol{A}_{i} \partial_{i} \boldsymbol{U}_{h}, \boldsymbol{V}_{h}\right)+\left(\boldsymbol{S} \boldsymbol{U}_{h}, \boldsymbol{V}_{h}\right) \\
& +\left(\boldsymbol{\tau} \mathcal{P}\left[\boldsymbol{F}-\left(\boldsymbol{\mu} \partial_{t} \boldsymbol{U}_{h}+\boldsymbol{A}_{i} \partial_{i} \boldsymbol{U}_{h}+\boldsymbol{S} \boldsymbol{U}_{h}\right)\right],-\boldsymbol{A}_{i} \partial_{i} \boldsymbol{V}_{h}+\boldsymbol{S}^{\top} \boldsymbol{V}_{h}\right)=\left(\boldsymbol{F}, \boldsymbol{V}_{h}\right) .
\end{aligned}
$$

Note that we have used the fact that the convective matrices $\boldsymbol{A}_{i}$ are symmetric in the above expression. It is also to be remarked that the matrix of stabilization parameters $\boldsymbol{\tau}$ is to be computed elementwise. With regard to the projection operator $\mathcal{P}$ different options exist. In the more classical ASGS (Algebraic Subgrid Scale) formulation, the projection is taken as 
the identity matrix over the space of finite element residuals, i.e., $\mathcal{P}=\boldsymbol{I}$. Alternatively, in the OSS (Orthogonal Subgrid Scale) method the subscales are assumed to be orthogonal to the finite element space and consequently, $\mathcal{P}=\boldsymbol{I}-\Pi_{h}$, with $\Pi_{h}$ standing for the $L^{2}$-projection onto the former $[25,35]$.

\subsection{Dimensional rescaling considerations}

To complete the stabilized formulation in (12), the crucial step of finding an appropriate expression for the stabilization matrix $\tau$ remains to be done. However, before addressing such an issue some considerations are to be made concerning the scalar products in the variational formulations (5) and (12). The r.h.s (right hand side) of these equations involves products of the type $\boldsymbol{U}^{\top} \boldsymbol{F}=p^{a} Q+u_{1}^{a} f_{1}+u_{2}^{a} f_{2}+u_{3}^{a} f_{3}$ (see (5)). If we use [.] to denote a dimensional group, it becomes necessary that $\left[p^{a} Q\right]=\left[u_{i}^{a} f_{i}\right]$. From the dimensions of the terms in the r.h.s of (3) it can readily be checked that $\left[p^{a} Q\right]=\left[u_{i}^{a} f_{i}\right]=M L T^{-3}$, with $M, L$ and $T$ standing for dimensions of mass, length and time respectively. Yet, when deriving an appropriate expression for $\boldsymbol{\tau}$ in the next subsection we will have to deal with products like $\boldsymbol{F}^{\top} \boldsymbol{F}$ or $\boldsymbol{U}^{\top} \boldsymbol{U}$, which are not dimensionally well defined. For instance, $\boldsymbol{F}^{\top} \boldsymbol{F}=Q^{2}+f_{1}^{2}+f_{2}^{2}+f_{3}^{2}$ but $\left[Q^{2}\right] \neq\left[f_{i}^{2}\right]$. Similarly, $\boldsymbol{U}^{\top} \boldsymbol{U}=p^{a 2}+u_{1}^{a 2}+u_{2}^{a 2}+u_{3}^{a 2}$ but again $\left[p^{a 2}\right] \neq\left[u_{i}^{a 2}\right]$. Consequently, and as quoted in $[26,31]$, it becomes necessary to rescale the original differential equations for these products to make sense.

This is tantamount to introducing the weighting matrix $\boldsymbol{M}$ (see [26]),

$$
\boldsymbol{M}=\left(\begin{array}{cccc}
m_{p} & 0 & 0 & 0 \\
0 & m_{\boldsymbol{u}} & 0 & 0 \\
0 & 0 & m_{\boldsymbol{u}} & 0 \\
0 & 0 & 0 & m_{\boldsymbol{u}}
\end{array}\right), \quad m_{p}:=\sqrt{\mu_{\boldsymbol{u}} / \mu_{p}}=\rho_{0} c_{0}, \quad m_{\boldsymbol{u}}:=\sqrt{\mu_{p} / \mu_{\boldsymbol{u}}}=\frac{1}{\rho_{0} c_{0}}
$$

to define the weighted scalar product $\boldsymbol{F}^{\top} \boldsymbol{M} \boldsymbol{F} \equiv|\boldsymbol{F}|_{M}^{2}$ which can be shown to be dimensionally correct given that $\left[m_{p} Q^{2}\right]=\left[m_{\boldsymbol{u}} f_{i}^{2}\right]=M L^{-2} T^{-3}$. Analogously, the inverse of $\boldsymbol{M}$ can be used to correctly define the product $\boldsymbol{U}^{\top} \boldsymbol{M}^{-1} \boldsymbol{U} \equiv|\boldsymbol{U}|_{M^{-1}}^{2}$. That is so because $\left[m_{p}^{-1} p^{a 2}\right]=\left[m_{\boldsymbol{u}}^{-1} u_{i}^{a 2}\right]=M T^{-3}$. Therefore, $\boldsymbol{M}$ and $\boldsymbol{M}^{-1}$ are the appropriate weighthing matrices to define products between any two force vectors $\boldsymbol{F}_{1}^{\top} \boldsymbol{M} \boldsymbol{F}_{2}$ and unknowns $\boldsymbol{U}_{1}^{\top} \boldsymbol{M}^{-1} \boldsymbol{U}_{2}$. We can also define an $\boldsymbol{M}$-weighted product between two arbitrary matrices $\boldsymbol{B}_{1}$ and $\boldsymbol{B}_{2}$ as $\boldsymbol{B}_{1}^{\top} \boldsymbol{M} \boldsymbol{B}_{2}$. The squared $\boldsymbol{M}$-pointwise norm of a matrix $\boldsymbol{B}$ will be given by $|\boldsymbol{B}|_{\boldsymbol{M}^{2}}^{2}=\sup \left\{\boldsymbol{X}^{\top} \boldsymbol{B}^{\top} \boldsymbol{M} \boldsymbol{B} \boldsymbol{X}\right\}, \forall \boldsymbol{X} ;|\boldsymbol{X}|_{\boldsymbol{M}^{-1}}=1$. For regular enough force vector functions it is also possible to introduce the following vector function $\boldsymbol{M}$-weighted scalar products,

$$
(\boldsymbol{U}, \boldsymbol{F})=\int_{\Omega} \boldsymbol{U}^{\top} \boldsymbol{F} d \Omega,\left(\boldsymbol{F}_{1}, \boldsymbol{F}_{2}\right)_{\boldsymbol{M}}:=\int_{\Omega} \boldsymbol{F}_{1}^{\top} \boldsymbol{M} \boldsymbol{F}_{2} d \Omega,\left(\boldsymbol{U}_{1}, \boldsymbol{U}_{2}\right)_{\boldsymbol{M}^{-1}}:=\int_{\Omega} \boldsymbol{U}_{1}^{\top} \boldsymbol{M}^{-1} \boldsymbol{U}_{2} d \Omega,
$$

whose induced norms are designated by $\|\cdot\|,\|\cdot\|_{M}$ and $\|\cdot\|_{M^{-1}}$.

\subsection{The matrix $\boldsymbol{\tau}$ of stabilization parameters}

A fruitful way to derive an expression for the matrix of stabilization parameters $\tau$ is from the spatial Fourier transform of the subscale equation (11). To that purpose we shall assume that the mean flow velocity and its derivatives are smoothly varying in the size of a typical mesh element, $\Omega_{e}$, so that the matrices $\boldsymbol{A}_{i}$ and $\boldsymbol{S}$ can be assumed constant within $\Omega_{e}$. We can thus define the Fourier transform of an arbitrary function $\boldsymbol{f}$ within $\Omega_{e}$ as $\hat{\boldsymbol{f}}(\boldsymbol{k}):=\int_{\Omega_{e}} \boldsymbol{f}(\boldsymbol{x}) \exp [-i(\boldsymbol{k} \cdot \boldsymbol{x}) / h] d \Omega_{e}$ (see e.g., [25]). Hereafter, a hat symbol will stand for Fourier transformed quantities and $k_{i}$ will denote the $i$-th component of the wavenumber adimensionalized by the characteristic mesh size $h$. Identifying the linear operator $\mathscr{L}:=\boldsymbol{A}_{i} \partial_{i}+\boldsymbol{S}$, we can express equation (11) in the wavenumber domain as,

$$
\hat{\mathscr{L}}(\boldsymbol{k}) \hat{\boldsymbol{U}}^{\prime}=-\mathrm{i} \frac{1}{h} k_{j} \boldsymbol{A}_{j} \hat{\boldsymbol{U}}^{\prime}+\boldsymbol{S} \hat{\boldsymbol{U}}^{\prime}=\hat{\boldsymbol{R}}_{h},
$$


with squared $\boldsymbol{M}$-norm

$$
\hat{\boldsymbol{U}}^{\prime \top} \hat{\mathscr{L}}(\boldsymbol{k})^{\top} \boldsymbol{M} \hat{\mathscr{L}}(\boldsymbol{k}) \hat{\boldsymbol{U}}^{\prime}=\hat{\boldsymbol{U}}^{\prime \top}\left(\frac{1}{h^{2}} k_{l} k_{j} \boldsymbol{A}_{l}^{\top} \boldsymbol{M} \boldsymbol{A}_{j}+2 \frac{\mathrm{i}}{h} k_{l}\left[\boldsymbol{A}_{l} \boldsymbol{M} \boldsymbol{S}\right]_{A}+\boldsymbol{S}^{\top} \boldsymbol{M} \boldsymbol{S}\right) \hat{\boldsymbol{U}}^{\prime}=\hat{\boldsymbol{R}}_{h}^{\top} \boldsymbol{M} \hat{\boldsymbol{R}}_{h} .
$$

Here, $\left[\boldsymbol{A}_{l} \boldsymbol{M} \boldsymbol{S}\right]_{A}$ stands for the skew-symmetric part of $\boldsymbol{A}_{l}^{\top} \boldsymbol{M} \boldsymbol{S}$. The induced norm for the residual (see (14)) fulfills

$$
\begin{aligned}
& \left\|\hat{\boldsymbol{R}}_{h}\right\|_{\boldsymbol{M}^{2}}^{2}=\int\left|\hat{\boldsymbol{R}}_{h}\right|_{\boldsymbol{M}^{2}}^{2} d \boldsymbol{k}=\int\left|\hat{\mathscr{L}}(\boldsymbol{k}) \hat{\boldsymbol{U}}^{\prime}\right|_{\boldsymbol{M}^{2}}^{2} d \boldsymbol{k} \leq \int|\hat{\mathscr{L}}(\boldsymbol{k})|_{\boldsymbol{M}}^{2}\left|\hat{\boldsymbol{U}}^{\prime}\right|_{\boldsymbol{M}^{-1}}^{2} d \boldsymbol{k} \\
& =\left|\hat{\mathscr{L}}\left(\boldsymbol{k}^{0}\right)\right|_{\boldsymbol{M}}^{2} \int\left|\hat{\boldsymbol{U}}^{\prime}\right|_{\boldsymbol{M}^{-1}}^{2} d \boldsymbol{k}=\left|\hat{\mathscr{L}}\left(\boldsymbol{k}^{0}\right)\right|_{\boldsymbol{M}}^{2}\left\|\hat{\boldsymbol{U}}^{\prime}\right\|_{\boldsymbol{M}^{-1}}^{2} \\
& =\rho\left(\hat{\mathscr{L}}\left(\boldsymbol{k}^{0}\right)^{\top} \boldsymbol{M} \hat{\mathscr{L}}\left(\boldsymbol{k}^{0}\right)\right)\left\|\hat{\boldsymbol{U}}^{\prime}\right\|_{\boldsymbol{M}^{-1}}^{2} \\
& \leq\left[\rho\left(\frac{1}{h^{2}} k_{l}^{0} k_{j}^{0} \boldsymbol{A}_{l}^{\top} \boldsymbol{M} \boldsymbol{A}_{j}\right)+\rho\left(2 \frac{\mathrm{i}}{h} k_{l}^{0}\left[\boldsymbol{A}_{l} \boldsymbol{M} \boldsymbol{S}\right]_{A}\right)+\rho\left(\boldsymbol{S}^{\top} \boldsymbol{M} \boldsymbol{S}\right)\right]\left\|\hat{\boldsymbol{U}}^{\prime}\right\|_{\boldsymbol{M}^{-1}}^{2},
\end{aligned}
$$

where the existence of a value of $\boldsymbol{k}^{0}$ that fulfils the equality in the second line is guaranteed by the mean value theorem. In the third line we use the fact that the squared $M$-pointwise norm of the operator $\mathscr{L}\left(\boldsymbol{k}^{0}\right)$ is given by its corresponding spectral radius $\rho\left(\hat{\mathscr{L}}\left(\boldsymbol{k}^{0}\right)^{\top} \boldsymbol{M} \hat{\mathscr{L}}\left(\boldsymbol{k}^{0}\right)\right)$. Herein, we understand $\rho(\boldsymbol{B})$ to denote the spectral radius of an arbitrary matrix $\boldsymbol{B}$ arising from the solution of the generalized eigenvalue problem $\boldsymbol{B} \boldsymbol{U}=\lambda \boldsymbol{M}^{-1} \boldsymbol{U}, \lambda$ being an eigenvalue. Finally, in the fourth line we use the fact that the spectral radius of the sum of two matrices is bounded by the summation of the matrices' individual spectral radii. All spectral radii in that line will be shown to be real (note that $\left[\boldsymbol{A}_{l} \boldsymbol{M} \boldsymbol{S}\right]_{A}$ is totally skew-symmetric and therefore has imaginary eigenvalues).

On the other hand, from the Fourier transform of the approximation for the subscales $\boldsymbol{U}^{\prime}=\boldsymbol{\tau} \boldsymbol{R}_{h}$, we can get the bound

$$
\left\|\hat{\boldsymbol{R}}_{h}\right\|_{\boldsymbol{M}}^{2} \leq\left|\boldsymbol{\tau}^{-1}\right|_{\boldsymbol{M}}^{2}\left\|\hat{\boldsymbol{U}}^{\prime}\right\|_{\boldsymbol{M}^{-1}}^{2} .
$$

The combination of (17) and (18) provides one way to find an expression for the stabilization matrix given that

$$
\rho\left(\boldsymbol{\tau}^{-1} \boldsymbol{M} \boldsymbol{\tau}^{-1}\right) \leq \rho\left(\frac{1}{h^{2}} k_{l}^{0} k_{j}^{0} \boldsymbol{A}_{l}^{\top} \boldsymbol{M} \boldsymbol{A}_{j}\right)+\rho\left(2 \frac{\mathrm{i}}{h} k_{l}^{0}\left[\boldsymbol{A}_{l} \boldsymbol{M} \boldsymbol{S}\right]_{A}\right)+\rho\left(\boldsymbol{S}^{\top} \boldsymbol{M S}\right) .
$$

Bounds for the spectral radii in (19) are provided in Appendix A. From (19) and (A.14) it follows

$$
\rho\left(\boldsymbol{\tau}^{-1} \boldsymbol{M} \boldsymbol{\tau}^{-1}\right) \leq \frac{1}{h^{2}}\left(C_{1}+C_{2} M\right)^{2}+\frac{1}{h} \frac{C_{3}}{c_{0}}|\nabla \overline{\boldsymbol{u}}|_{F}+\frac{C_{4}}{c_{0}^{2}}|\nabla \overline{\boldsymbol{u}}|_{F}^{2} .
$$

Taking into account that $|\nabla \overline{\boldsymbol{u}}| \sim \mathcal{O}(\overline{\boldsymbol{u}} / L)<<\overline{\boldsymbol{u}} / h$, with $L$ standing for a characteristic lenght of the domain, and redefining the constants in the expressions hereafter where appropriate, we can check that the bound on the $\boldsymbol{M}$-norm of the stabilization matrix behaves as

$$
\rho\left(\boldsymbol{\tau}^{-1} \boldsymbol{M} \boldsymbol{\tau}^{-1}\right)<<\frac{1}{h^{2}}\left[C_{1}+C_{2} \mathcal{O}(M)+C_{3} \mathcal{O}\left(M^{2}\right)\right] .
$$

Note that the discarded term involving $M^{2}$ in (A.8)-(A.9) would have given place to a term $\sim \mathcal{O}\left(M^{3}\right)$ in $(21)$.

In order to get a simple expression for the stabilization matrix we may take it to be diagonal, $\boldsymbol{\tau}=\operatorname{diag}\left(\tau_{p}, \tau_{\boldsymbol{u}}, \tau_{\boldsymbol{u}}\right)$. Given that the scaling matrix $\boldsymbol{M}$ in (13) is also diagonal, it follows that

$$
\operatorname{Spec}_{\boldsymbol{M}^{-1}}\left(\boldsymbol{\tau}^{-1} \boldsymbol{M} \boldsymbol{\tau}^{-1}\right)=\left\{\left(\frac{m_{p}}{\tau_{p}}\right)^{2},\left(\frac{m_{\boldsymbol{u}}}{\tau_{\boldsymbol{u}}}\right)^{2},\left(\frac{m_{\boldsymbol{u}}}{\tau_{\boldsymbol{u}}}\right)^{2}\right\} .
$$


From (20) and the subsequent observation (21), it becomes clear that what we need from the stabilization parameter is to provide a term depending on the mesh size like in the standard mixed wave equation with no convection [26], a term to control the flow local mean velocity $\overline{\boldsymbol{u}}$, and a term that controls the mean velocity derivatives through $|\nabla \overline{\boldsymbol{u}}|_{F}^{2}$. To get a rather simple expression for the parameter fulfilling these requisites, our proposal is to equal every eigenvalue of $\boldsymbol{\tau}^{-1} \boldsymbol{M} \boldsymbol{\tau}^{-1}$ in (22) to a term of the type $\left[h^{-2}\left(C_{1}+C_{2} \overline{\boldsymbol{u}} / c_{0}\right)^{2}+\left(C_{3}|\nabla \overline{\boldsymbol{u}}|_{F} / c_{0}\right)^{2}\right]$. Direct generalization to the three dimensional case results in

$$
\begin{gathered}
\boldsymbol{\tau}=\left(\begin{array}{cccc}
\tau_{p} & 0 & 0 & 0 \\
0 & \tau_{\boldsymbol{u}} & 0 & 0 \\
0 & 0 & \tau_{\boldsymbol{u}} & 0 \\
0 & 0 & 0 & \tau_{\boldsymbol{u}}
\end{array}\right) \text { with, } \\
\tau_{p}=\frac{\rho_{0} c_{0}^{2} h}{\tau_{\boldsymbol{u}}=\frac{h}{\left.\left.\rho_{0}\left[\left(c_{0} C_{1}+C_{2}|\overline{\boldsymbol{u}}|\right)^{2}+\left(C_{3} h|\nabla \overline{\boldsymbol{u}}|_{F}\right)^{2}\right]_{1}+C_{2}|\overline{\boldsymbol{u}}|\right)^{2}+\left(C_{3} h|\nabla \overline{\boldsymbol{u}}|_{F}\right)^{2}\right]^{1 / 2}},}
\end{gathered}
$$

where use has been made of the expressions for $m_{p}$ and $m_{\boldsymbol{u}}$ in (13).

The matrix $\boldsymbol{\tau}$ of stabilization parameters (23) has to be inserted into (12) to get the final stabilized variational formulation proposed in this article. It only remains to find the values of the constants $C_{1}, C_{2}$ and $C_{3}$. This will be done by means of the numerical experiments in Section 4.1. As regards the constants, it should be pointed out that there is a certain tolerance for them, in the sense that one does not necessarily need to get their optimal values as long as they are able to provide enough stabilization. For a given mesh size $h$, the error may be larger or smaller depending on the particular values of the constants, but the slope of the convergence curves will remain unchanged for a wide range of values, which is what matters in fact.

Finally, note that in the case of no mean flow, $\overline{\boldsymbol{u}}=\mathbf{0}$, the stabilization parameters for the standard wave equation in mixed form are recovered,

$$
\tau_{p}=C h \sqrt{\mu_{\boldsymbol{u}} / \mu_{p}} \quad \tau_{\boldsymbol{u}}=C h \sqrt{\mu_{p} / \mu_{\boldsymbol{u}}},
$$

with $C$ standing for a constant (see [26]). Likewise, if there was no reaction term, $\boldsymbol{S}=\mathbf{0}$, the factors $|\nabla \overline{\boldsymbol{u}}|_{F}^{2}$ would have not appeared in (23), and we would have recovered analogous stabilization parameters to those found for the standard wave equation in mixed form in an ALE framework [32], or for the equations of wave propagation in shallow waters [31].

\subsection{Fully discrete problem}

In the above developments, the time variable has been left continuous. To proceed to the time discretization we have equally split the time interval $\left[\begin{array}{ll}0 & T\end{array}\right]$ into $N$ steps $0<$ $t^{1}<t^{2}<\ldots<t^{n}<\ldots<t^{N} \equiv T$ with $\Delta t:=t^{n+1}-t^{n}$ denoting the time step size. Hereafter, $g^{n}$ will stand for the evaluation of a time dependent function $g(t)$ at $t^{n}=n \Delta t$. Given that we aim at computing the aeroacoustic field only at a few wavelengths form the acoustic source region, a second order backward differentiation formula (BDF2) will prove accurate enough for the time discretization of (12). Was it necessary to compute the acoustic field at several tenth or hundred wavelengths from the source, a less dissipative numerical scheme like the Crank-Nicholson or a higher-order one would prove better. Identifying $\delta_{t} g^{n+1}:=(1 / 2 \Delta t)\left(3 g^{n+1}-4 g^{n}+g^{n-1}\right)$ allows one to write the time discrete version of (12) as

$$
\begin{aligned}
& \left(\boldsymbol{\mu} \delta_{t} \boldsymbol{U}_{h}^{n+1}, \boldsymbol{V}_{h}\right)+\left(\boldsymbol{A}_{i} \partial_{i} \boldsymbol{U}_{h}^{n+1}, \boldsymbol{V}_{h}\right)+\left(\boldsymbol{S} \boldsymbol{U}_{h}^{n+1}, \boldsymbol{V}_{h}\right) \\
& +\left(\boldsymbol{\tau} \mathcal{P}\left[\boldsymbol{F}^{n+1}-\left(\delta_{t} \boldsymbol{U}_{h}^{n+1}+\boldsymbol{A}_{i} \partial_{i} \boldsymbol{U}_{h}^{n+1}+\boldsymbol{S} \boldsymbol{U}_{h}^{n+1}\right)\right],-\boldsymbol{A}_{i} \partial_{i} \boldsymbol{V}_{h}+\boldsymbol{S}^{\top} \boldsymbol{V}_{h}\right)=\left(\boldsymbol{F}^{n+1}, \boldsymbol{V}_{h}\right) .
\end{aligned}
$$




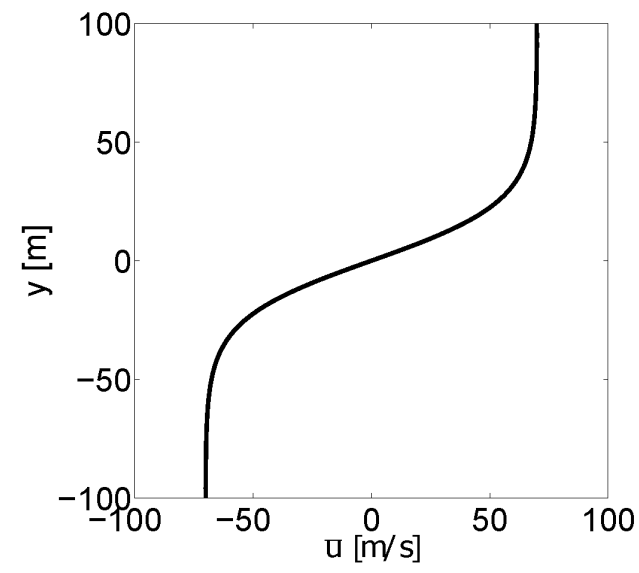

(a)

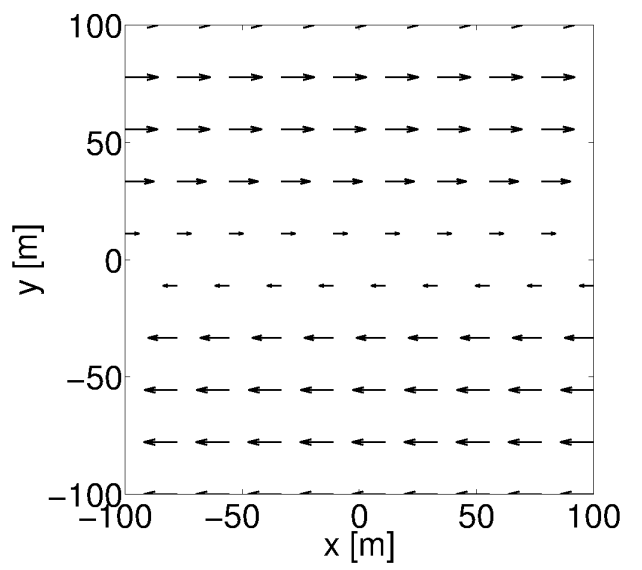

(b)

Figure 1. (a) Shear mean velocity profile and (b) resulting vector field for Cases 3 and 4.

If we choose the OSS approach for the stabilized spatial discretization it will follow that $\mathcal{P}\left(\delta_{t} \boldsymbol{U}_{h}\right)=0$. This is so because $U_{h}$ belongs to the finite element space and we are projecting $U_{h}$ onto the subscale space, which is precisely orthogonal to the latter space (remember that $\left.\mathcal{P}=\boldsymbol{I}-\Pi_{h}\right)$. The expanded fully discrete problem in time and space that results from accounting for (5) in (25) and that has been implemented for the numerical examples in the forthcoming section reads

$$
\begin{aligned}
& \frac{1}{\rho_{0} c_{0}^{2}}\left(\delta_{t} p_{h}^{a^{n+1}}, q_{h}\right)+\frac{1}{\rho_{0} c_{0}^{2}}\left(\overline{\boldsymbol{u}} \cdot \nabla p_{h}^{a^{n+1}}, q_{h}\right)+\left(\nabla \cdot \boldsymbol{u}_{h}^{a^{n+1}}, q_{h}\right) \\
& +\left(\tau_{p} \mathcal{P}\left[Q^{n+1}-\frac{1}{\rho_{0} c_{0}^{2}} \overline{\boldsymbol{u}} \cdot \nabla p_{h}^{a^{n+1}}-\nabla \cdot \boldsymbol{u}_{h}^{a^{n+1}}\right],-\frac{1}{\rho_{0} c_{0}^{2}} \overline{\boldsymbol{u}} \cdot \nabla q_{h}-\nabla \cdot \boldsymbol{v}_{h}\right)=\left(Q^{n+1}, q_{h}\right), \\
& \rho_{0}\left(\delta_{t} \boldsymbol{u}_{h}^{a^{n+1}}, \boldsymbol{v}_{h}\right)+\rho_{0}\left(\overline{\boldsymbol{u}} \cdot \nabla \boldsymbol{u}_{h}^{a^{n+1}}, \boldsymbol{v}_{h}\right)+\left(\nabla p_{h}^{a^{n+1}}, \boldsymbol{v}_{h}\right)+\rho_{0}\left(\boldsymbol{u}_{h}^{a^{n+1}} \cdot \nabla \overline{\boldsymbol{u}}, \boldsymbol{v}_{h}\right) \\
& +\left(\tau_{\boldsymbol{u}} \mathcal{P}\left[-\rho_{0} \overline{\boldsymbol{u}} \cdot \nabla \boldsymbol{u}_{h}^{a^{n+1}}-\nabla p_{h}^{a^{n+1}}-\rho_{0} \boldsymbol{u}_{h}^{a^{n+1}} \cdot \nabla \overline{\boldsymbol{u}}\right],-\rho_{0} \overline{\boldsymbol{u}} \cdot \nabla \boldsymbol{v}_{h}-\nabla q_{h}+\rho_{0} \boldsymbol{v}_{h} \cdot \nabla \overline{\boldsymbol{u}}\right)=0 .
\end{aligned}
$$

Finally, note that in order to get the final finite element matrix algebraic system we simply need to substitute $p_{h}=\sum_{a=1}^{n_{p}} N_{p}^{a} P^{a}$, and $\boldsymbol{u}_{h}=\left(\sum_{b=1}^{n_{u}} N_{u}^{b} U_{x}^{b}, \sum_{b=1}^{n_{u}} N_{u}^{b} U_{y}^{b}, \sum_{b=1}^{n_{u}} N_{u}^{b} U_{z}^{b}\right)^{\top}$, and their analogous for the test functions $q_{h}$ and $\boldsymbol{v}_{h}$ in (26). In the preceding expressions $n_{p}$ and $n_{u}$ respectively stand for the total number of pressure and velocity nodes in the domain. $P^{a}$ denote the unknown nodal pressure values and $U_{x}^{b}, U_{y}^{b}, U_{z}^{b}$ the unknown nodal velocity component values. $N_{p}^{a}$ are the pressure shape functions and $N_{u}^{b}$ the velocity shape functions, which are taken equal in the forthcoming section on numerical examples.

\section{NUMERICAL EXAMPLES}

\subsection{Wave propagation in solenoidal convective and shear mean flows}

The purpose of this example is twofold. On the one hand it will allow one to check the influence of the various terms appearing in the acoustic perturbation equations (3). On the other hand, it will help determining the values of the constants $C_{1}, C_{2}$ and $C_{3}$ appearing in the stabilization parameters of (23). Throughout section 4 , the units of all quantities will be those of the SI system and will be not explicitly written. 
The example consists of a squared computational domain having dimensions $\Omega=$ $[-100,100] \times[-100,100]$. A time varying monopole source of strength,

$$
Q=\exp \left[-\ln (2) \frac{x^{2}+y^{2}}{9}\right] \cos (\omega t)
$$

has been placed at the origin $(0,0)$ and generates acoustic waves of angular frequency $\omega=175$. The propagation of the acoustic waves will be analyzed for different solenoidal mean velocity profiles $\overline{\boldsymbol{u}}$ in the domain. That will reveal the importance of the distinct APE terms in (3). The cases that have been considered are:

- Case A.1: No mean flow, i.e., $\overline{\boldsymbol{u}}=\mathbf{0}$. In this case the APE reduce to the standard wave equation in mixed form.

- Case A.2: A constant mean flow with $\overline{\boldsymbol{u}}=(70,0)$. The APE now reduce to the convective wave equation in mixed form for uniform mean flows.

- Case A.3: A solenoidal shear mean flow with the profile plotted in Fig. 1a resulting from equation (28) below. This corresponds to the convective wave equation in mixed form for the case of a non-uniform mean flow. The reaction term $\rho_{0} \boldsymbol{u}^{a} \cdot \nabla \overline{\boldsymbol{u}}$ in (3b) is not taken into account in the simulation.

- Case A.4: The shear mean flow of Case 3 but now considering the full APE in (3) with all non-uniform convective and reaction terms included. This was the benchmark case proposed in [1] to test several APE formulations.

To perform all the simulations for the cases above, the computational domain has been discretized with an unstructured mesh of 87616 triangular elements. This has resulted in approximately nine elements per wavelength. Spurious reflections at the boundaries have been avoided by means of a simple perfectly matched layer (PML) according to the model in [38]. Two additional absorption terms $\alpha p$ and $\alpha^{*} \boldsymbol{u}$ have been respectively added to the equations (3a) and (3b). $\alpha$ is the attenuation coefficient and $\alpha^{*}:=\left(\mu_{\boldsymbol{u}} / \mu_{p}\right) \alpha$. The attenuation factor is set to zero within the computational domain $\Omega$, while it has been set to $\alpha=0.0004$ in the absorptive PML surrounding $\Omega$. The width of the PML is 50. With regard to the values of the physical parameters in (3), we have considered an air density of $\rho_{0}=1.14$ and a sound speed of $c_{0}=350$. The shear mean velocity for Cases A.3 and A.4 is defined by (see Fig. 1a),

$$
\overline{\boldsymbol{u}}(x, y)=(\bar{U} \tanh (2 y / \delta), 0)
$$

where $\bar{U}$ is the maximal velocity with value $\bar{U}=70$ (Mach number $M=0.2$ ) and $\delta=50$ denotes the shear-layer thickness. The resulting mean velocity vector field is plotted in Fig. $1 \mathrm{~b}$. In what concerns time evolution, an incremental step of $\Delta t=7 \times 10^{-4}$ has been chosen to discretize the interval $[0,0.5]$. Finally, the values $C_{1}=100, C_{2}=500$ and $C_{3}=100000^{2}$ have been taken for the constants in the stabilization parameter (see below for a justification on that choice). It is to be noted that the stabilization parameter dictates the critical time step, $\Delta t_{c r}=\rho_{0} \tau_{\boldsymbol{u}}=\left(\rho_{0} c_{0}^{2}\right)^{-1} \tau_{p}$ of the simulation [39]. This has local values because it depends on the characteristic mesh size, $h$, as well as on the mean velocity field $\overline{\boldsymbol{u}}$ and its gradient $\nabla \overline{\boldsymbol{u}}$, see (23). If we take the minimum value of $\triangle t_{c r}$ over the computational domain, this results in a CFL number of $C_{C F L}=\triangle t / \triangle t_{c r} \simeq 614$. Instead, if we take an averaged value for $\triangle t_{c r}$ over the domain we get $C_{C F L} \simeq 74$. This reflects nothing but the well-known fact that when using implicit methods one can use time step sizes significantly larger than the critical time step.

The resulting acoustic pressure fields for Cases A.1-A.4 have been plotted in Fig. 2 at the time instant $t=0.5$. As observed in Fig. 2a, the wavefronts simply consist of concentric circles propagating outwards for Case A.1, because there is no mean velocity in the domain. We thus recover the expected radiation pattern of a monopole source. When a uniform mean flow is imposed moving from left to right in Case A.2 (Fig. 2b), the front waves travelling 


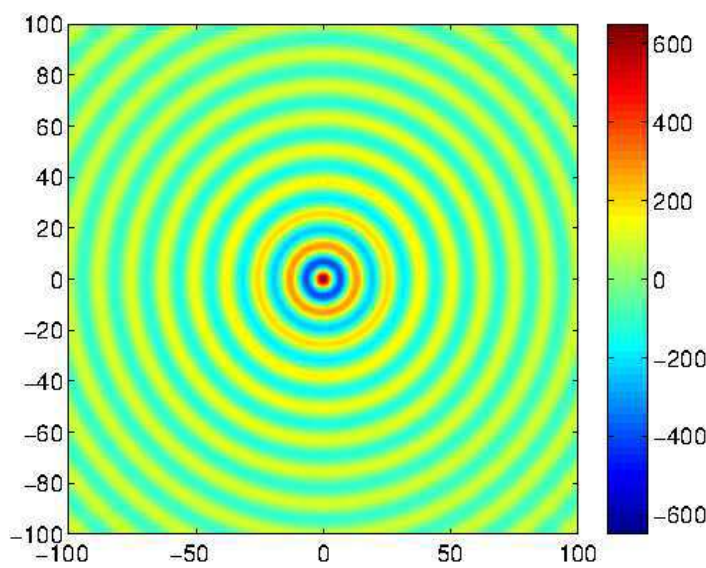

(a)

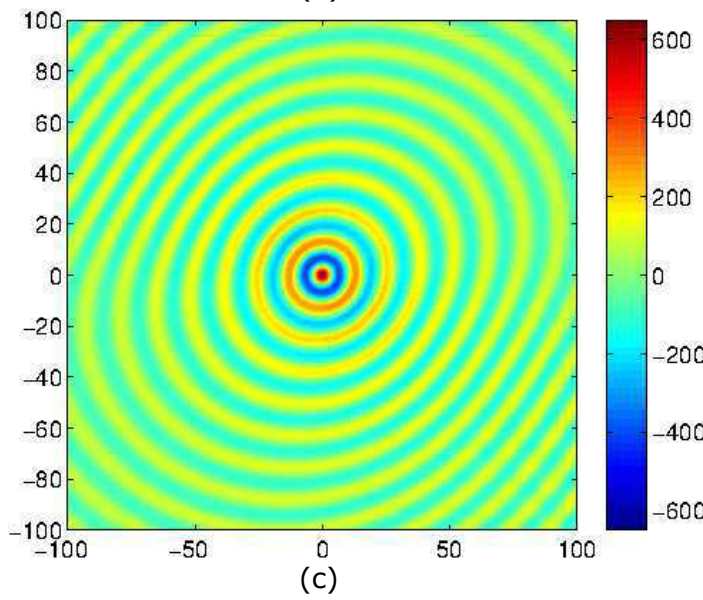

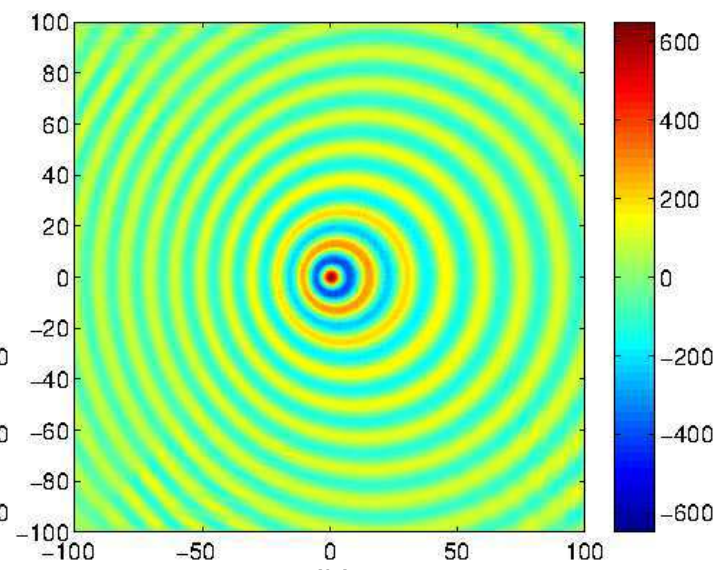

(b)

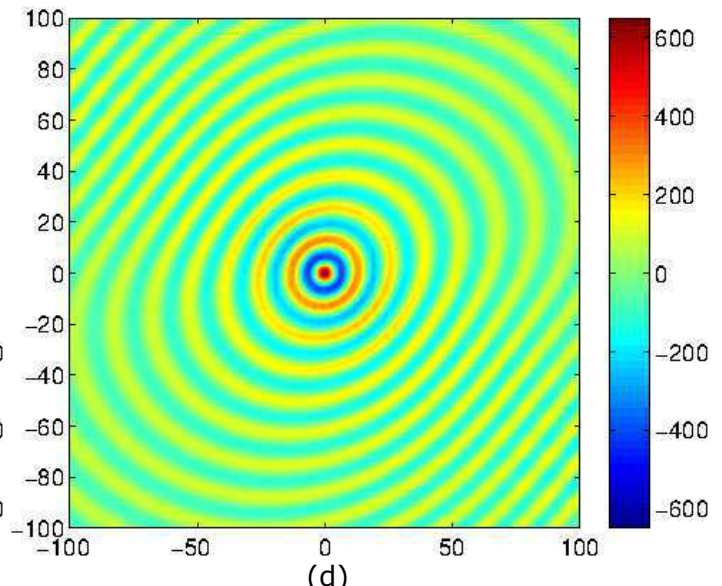

Figure 2. Acoustic pressure contours at time $t=0.5$. (a) Case A.1: No mean flow. (b) Case A.2: Uniform mean flow. (c) Case A.3: Shear flow with reaction term excluded. (d) Case A.4: Shear flow with reaction term included.

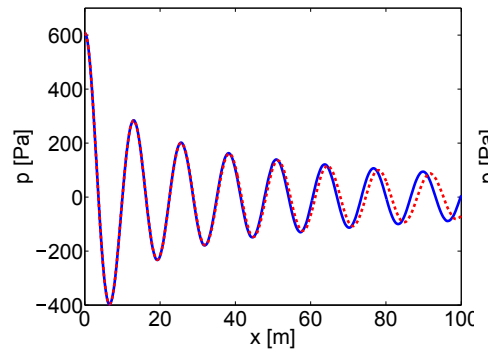

(a)

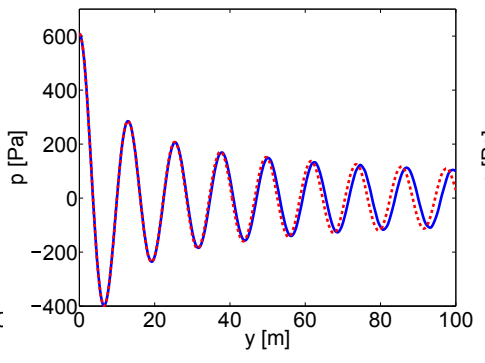

(b)

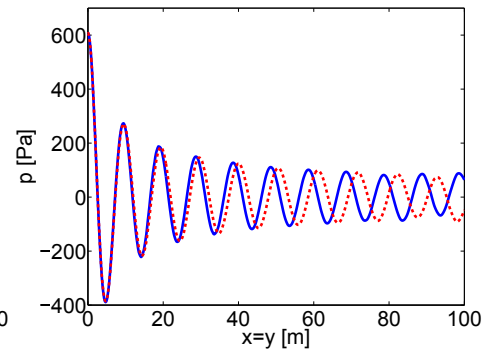

(c)

Figure 3. Pressure cuts at $t=0.5$ corresponding to Fig. 2. Case A.3 (dashed red), Case A.4: (continuous blue). (a) $x$ - axis. (b) $y$-axis. (c) $x=y$.

upstream get stretched and the wavelength diminishes, whereas the opposite effect takes place for waves propagating downstream. Cases A.3 and A.4 serve to show the importance of taking into account the reaction term in the APE. It is apparent from Figs. $2 \mathrm{c}$ and $d$ that if one was to simulate sound propagation in a shear flow, the use of a convective mixed wave equation with non-uniform velocity (Case A.3) would lead to substantial differences when compared to the predictions made by the full APE (Case A.4). These discrepancies might be better appreciated by plotting the acoustic pressure along the positive $x$-axis, positive 
$y$-axis and along a diagonal cut $x=y$ in the second quadrant of the subfigures Figs. 2c, $\mathrm{d}$ (see respectively Figs. 3a, b and c where the dashed red line stands for Case A.3 and the blue continuous line for Case A.4).

A plot showing the differences in wave propagation for Cases A.1-A.4 is that of Fig. 4. In that figure we see the time evolution of the acoustic pressure tracked at an arbitrary point A with coordinates $\boldsymbol{x}_{A}=(49.6,-34.1)$. The differences in amplitudes and phases between cases become again very visible. The zero pressure initial time steps correspond to the time it takes for the first wavefront to travel from the origin, where the source is placed, to A.

Further, it is worthwhile noticing that the APE equations offer not only the possibility to compute the acoustic pressure but also the acoustic particle velocity. In Fig. 5 we show the $x$ and $y$ components of the velocity field at time $t=0.5$, corresponding to the case A.4. As observed, the values of the velocity components are in clear agreement with the wavefront propagation directions in Fig. 2d.

On the other hand, as mentioned above, the second goal of this section is to determine the values of the constants $C_{1}, C_{2}$ and $C_{3}$ in the stabilization parameters of (23). The simulations just reported have been carried out following the numerical strategy in section 3.4 , but the values for that constants were yet to be presented. Let us remark that for Case A.1, $C_{1}$ is the only constant playing a role given that $\overline{\boldsymbol{u}}=\mathbf{0}$. For Cases A.2 and A.3, $C_{1}$ and $C_{2}$ get involved but not $C_{3}$ because the reaction term is not contemplated. Finally, all constants are to be considered in Case A.4.

To find suitable values for the constants, we have compared the results of the stabilized FEM simulations with those from a reference solution computed with a very fine mesh of 420585 elements. For such a fine mesh the contribution of the stabilization terms has been checked to be almost negligible. To proceed we have chosen to compute the relative $L^{2}$-error between the computed acoustic pressure $p^{a}$ and that from the reference solution $p^{r}$. This is given by $\varepsilon=\left\|p^{a}-p^{r}\right\|_{2} /\left\|p^{r}\right\|_{2}$ with $\left\|p^{a}-p^{r}\right\|_{2}=\left[\int_{\Omega_{\varepsilon}}\left(p^{a}-p^{r}\right)^{2} d \Omega_{\varepsilon}\right]^{1 / 2}$. The error is computed in the subdomain $\Omega_{\varepsilon} \subset \Omega$ so as not to contact the boundaries of $\Omega$ and avoid any possible pollution that could stem from the performance of the PML. It is to be noted that we could also have included the relative error for the acoustic velocity in the process, or a weighted combination of relative errors for both, the acoustic velocity and the acoustic pressure. However, given that usually in acoustic problems the latter is the main variable of interest, we have focused on the acoustic pressure.

The value of the $C_{1}$ constant was already established in [26], where it appeared in a slightly different form. Given that for $\overline{\boldsymbol{u}}=\mathbf{0}$ the results from [26] are to be recovered, we have fixed $C_{1}=100$ to that purpose. The constant $C_{2}$ has been selected focusing on Case A.3. For the

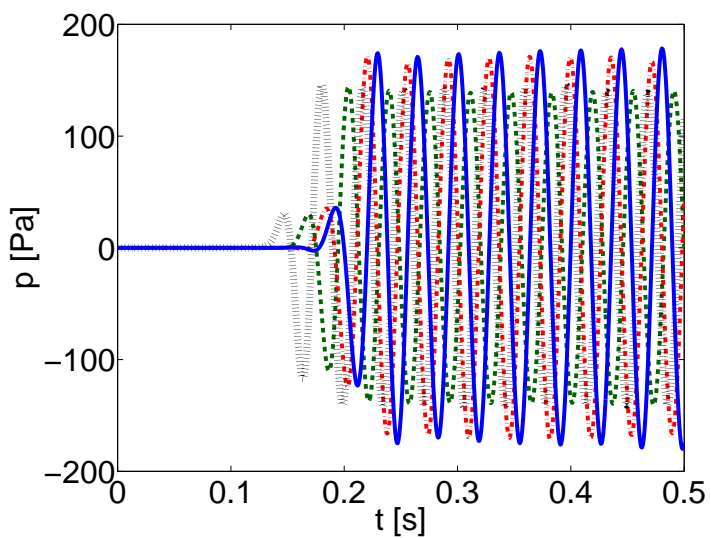

Figure 4. Comparison of the evolution of the pressure at point (49.6,-34.1) for Case A.1 (dashed green), Case A.2 (dotted black), Case A.3 (dot-dashed red) and Case A.4 (continuous blue). 


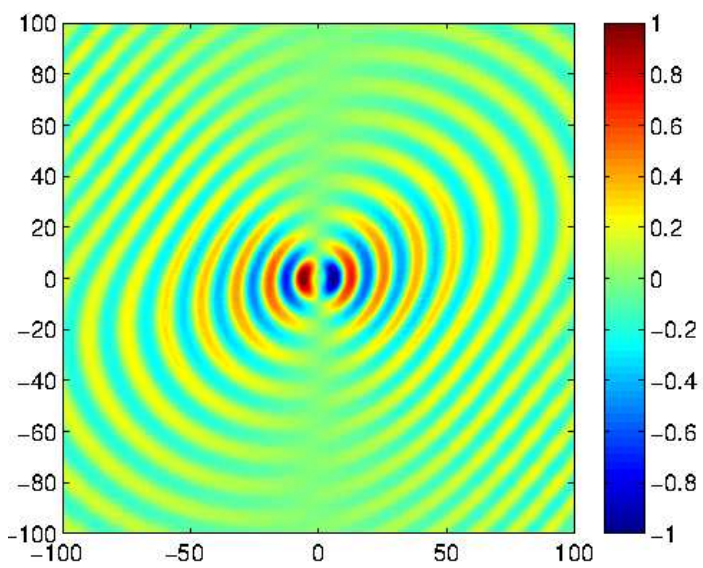

(a)

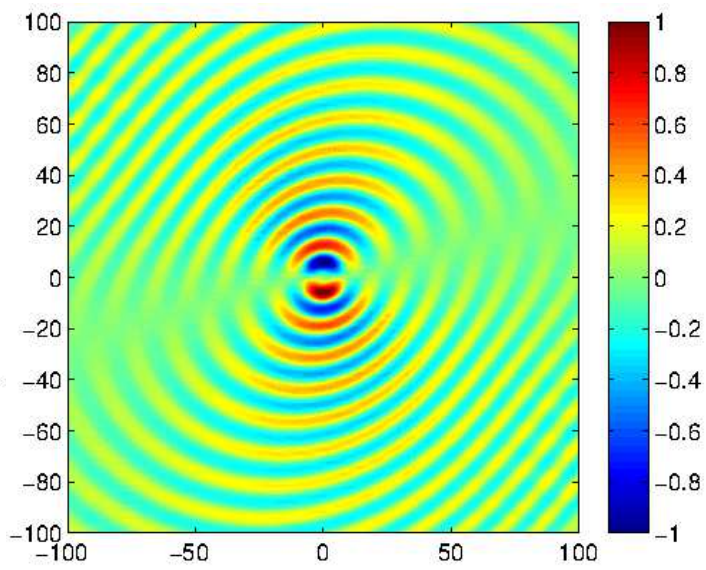

(b)

Figure 5. Acoustic velocity contours at time $t=0.5$ for Case A.4. (a) x-component (b) ycomponent.

terms in the denominator of (23) to have similar influence, $C_{2}$ should not surpass a value of 2000 . Having a look at the relative error plot in Fig. 6a we observe that an appropriate value for $C_{2}$ is $C_{2}=500$. Once fixed $C_{1}=100$ and $C_{2}=500$, we have resorted to Case A.4 to adjust the constant $C_{3}$. This constant should not exceed $150000^{2}$, and as observed from Fig. $6 \mathrm{~b}$, the relative error get stabilized for $C_{3} \approx 100000^{2}$. As in most stabilized numerical methods, once tuned, the constants $C_{1}=100, C_{2}=500$ and $C_{3}=100000^{2}$ are expected to have a rather general character and therefore to apply to many other numerical examples. This is so because the values of the constants do not significantly alter the convergence rate of the solutions when refining the computational mesh (see e.g., [26, 32] among others). The situation can be thought as being quite analogously to what happens when one changes one computational mesh for another. The overall error of the solution will be different in both meshes, yet the rate of convergence to the reference solution when refining them will be kept unaltered.

To conclude this example, we show the acoustic pressure for the stabilized FEM and reference solutions for the diagonal $x=y$ of the domain in Fig. 7. As observed, almost no

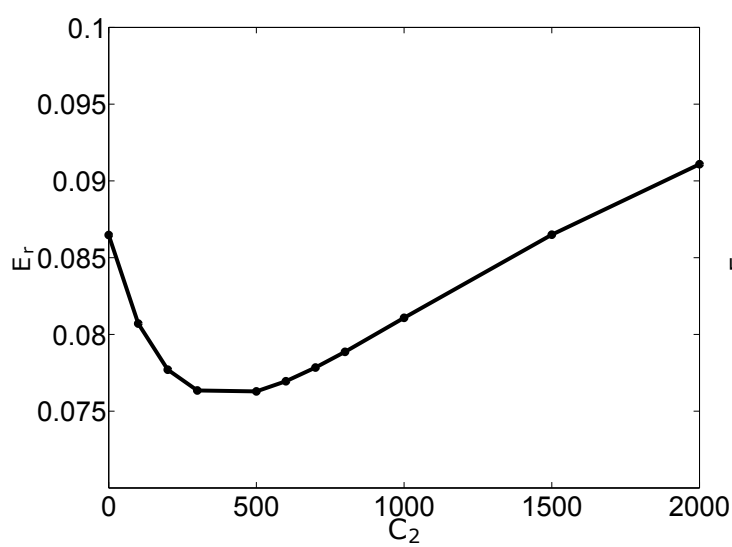

(a)

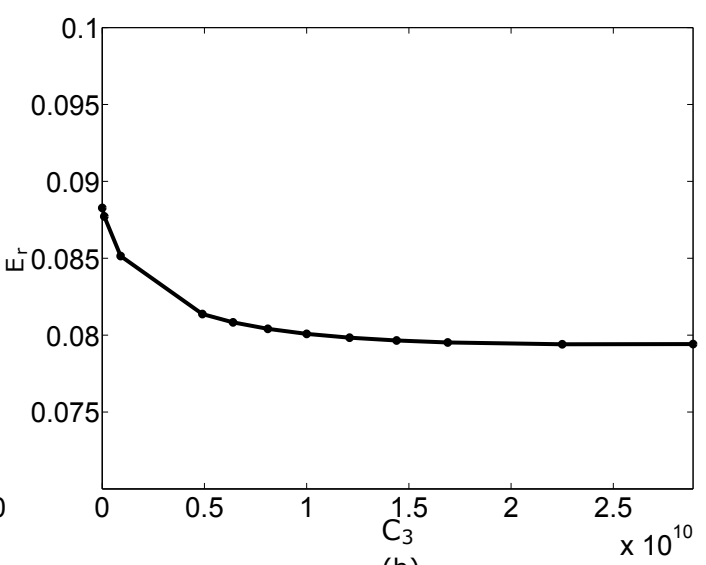

(b)

Figure 6. Relative error of the stabilized FEM formulation dependence on the values of the constants in the stabilization matrix. (a) Case 3: relative error dependence on $C_{2}$. (b) Case 4 : relative error dependence on $C_{3}$. 


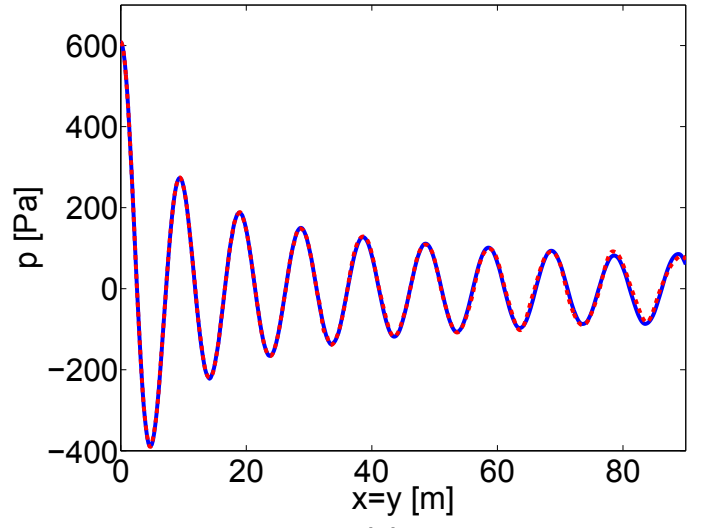

(a)

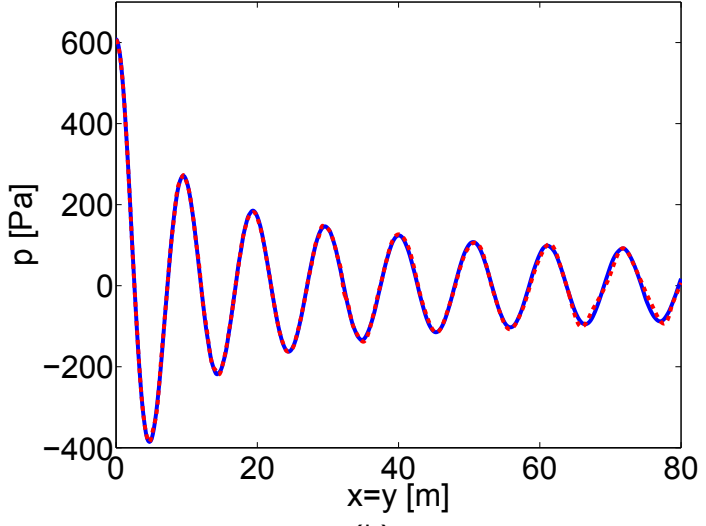

(b)

Figure 7. Reference acoustic pressure (blue line) versus stabilized FEM acoustic pressure (red dashed line) for the domain diagonal taking $C_{1}=100, C_{2}=500$ and $C_{3}=100000^{2}$. (a) Case A.3. (b) Case A.4.

differences can be appreciated. Yet, had we considered no stabilization at all, the Galerkin FEM solution would have simply blown up in finite time, as appreciated in Fig. 8 for the pressure evolution at point $\boldsymbol{x}_{A}=(49.6,-34.1)$ of the domain.

\subsection{Aeolian tone generated by a single cylinder}

As a final numerical test, we will apply the stabilized FEM approach to the APE, to a more complex situation. This consists in the classical problem of aeolian tone generation by flow past a cylinder. From a physical point of view, the aeroacoustic problem of aeolian tones is well-known and described in literature, see e.g., [40, 41] among many others. The problem consists of a flow impinging on a cylinder. For a certain range of Reynolds numbers, a wake of shedding vortices develops past the cylinder inducing lift fluctuating forces on it. That results in the emission of acoustic waves, which exhibit a dipolar radiation pattern at the acoustic far field. For low Mach numbers, the problem can be solved by first performing an incompressible CFD simulation to extract the acoustic source terms and the mean flow, which can then be inserted into the APE (3) to resolve the acoustic field. Our interest in this example is that of showing again the role played by the convection and reaction

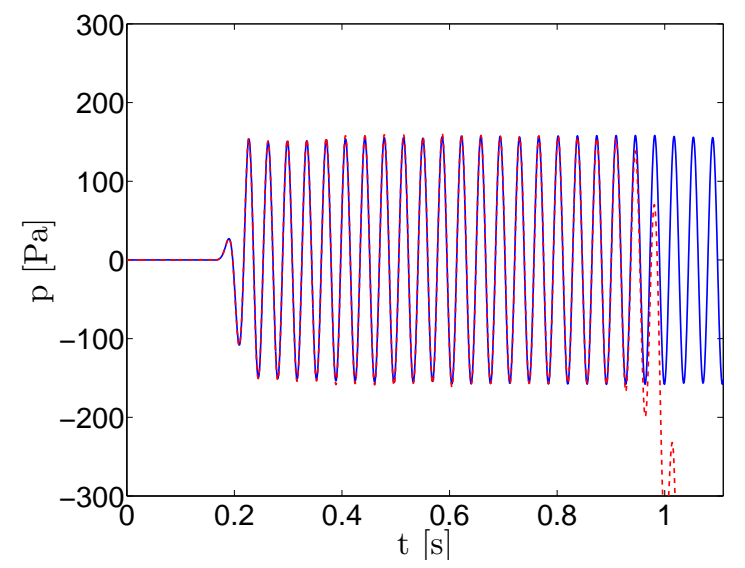

Figure 8. Comparison of the evolution of the pressure at point $(49.6,-34.1)$ for the non-stabilized Galerkin FEM solution (dashed red line) and the stabilized FEM solution (continuous blue line). The Galerkin FEM solution blows up. 

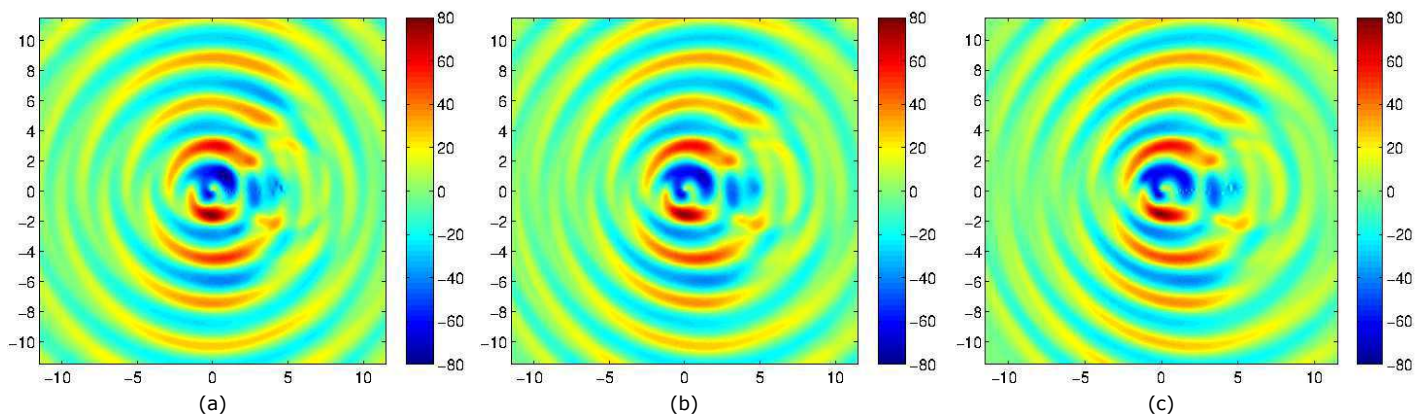

Figure 9. Acoustic pressure contours at time $t=0.15$. (a) Case C.1. (b) Case C.2. (c) Case C.3.

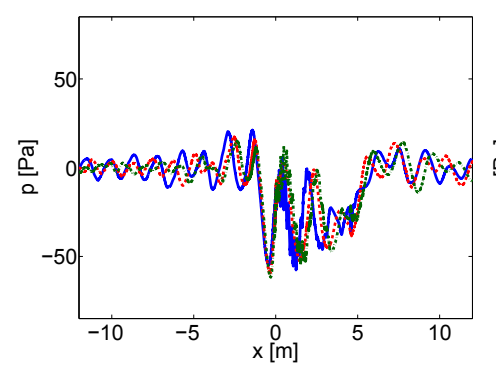

(a)

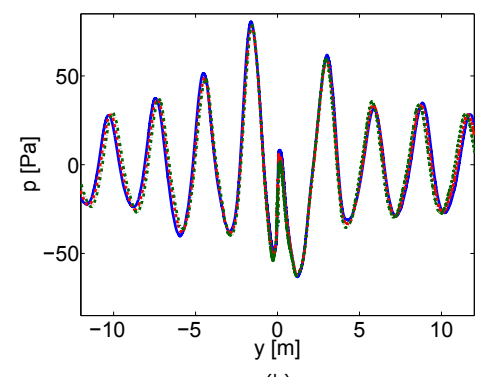

(b)

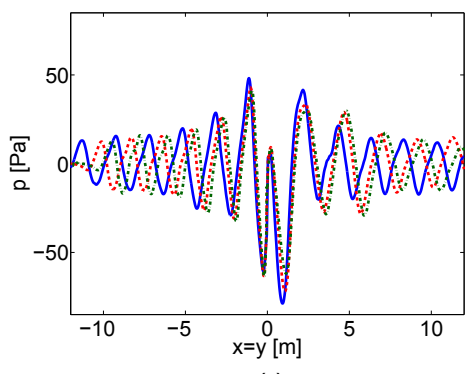

(c)

Figure 10. Pressure cuts at $t=0.15$ corresponding to Fig. 9. Case C.1 (continuous blue), Case C.2 (dashed red line), Case C.3 (dashed-dot green line). (a) $x$ - axis. (b) $y$ - axis. (c) $x=y$.

terms of the APE in the generated aeroacoustic pressure. Consequently, no results will be presented concerning the CFD simulation (they are rather standard and can be found elsewhere e.g., $[17,14])$.

In a nutshell and as regards the CFD computation, a two-dimensional cylinder with diameter $\mathrm{D}=0.1$ has been embedded in a squared computational domain of dimensions $\Omega_{C F D}=[-6,6] \times[-6,6]$ with an impinging flow velocity, in Cartesian coordinates, of $\boldsymbol{u}_{0}=(50,0)$ (Mach number $M \approx 0.14$ for a sound speed of $c_{0}=350$ ). The Reynolds number of the problem is $\operatorname{Re}=\rho_{0}\left|\boldsymbol{u}_{0}\right| D / \mu=1000$, where $\mu$ denotes the air viscosity. The domain $\Omega_{C F D}$ has been meshed with 295141 linear finite elements. The incompressible Navier-Stokes equations have been solved for a time range of 0.15, using the second order BDF2 time marching scheme with a time step of $9 \times 10^{-5}$. The solution has resulted in the generation of a Von Kármán vortex street, with vortices shed at a frequency of 120.
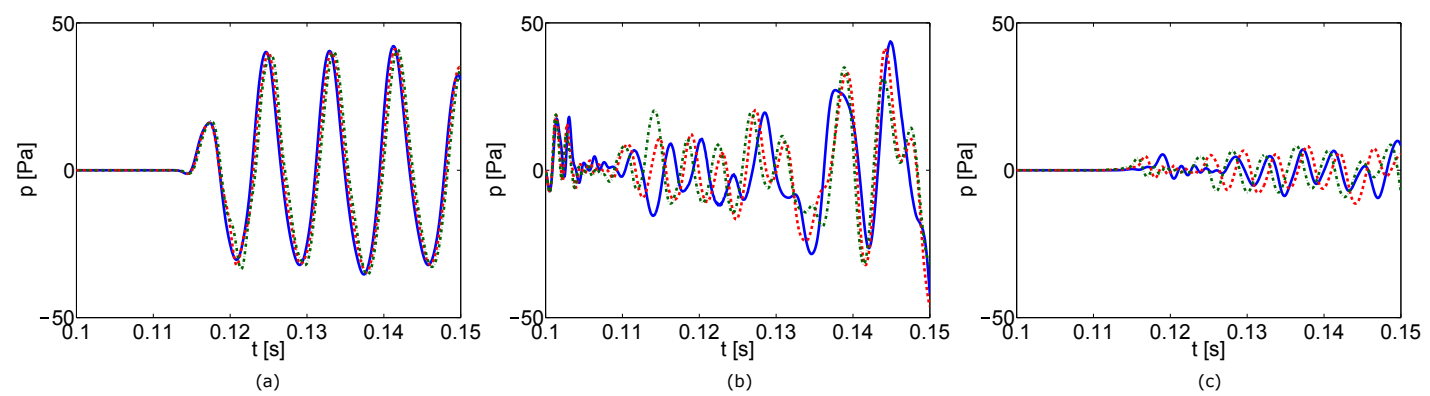

Figure 11. Comparison of the evolution of the pressure for Case C.1 (continuous blue), Case C.2 (dashed red line) and Case C.3 (dashed-dot green line). (a) $\boldsymbol{x}_{1}=(-0.2,5.8)$. (b) $\boldsymbol{x}_{2}=(3.1,0)$. (c) $\boldsymbol{x}_{3}=(9,0)$. 
The APE have been resolved in a larger computational domain $\Omega_{a c}=[-12,12] \times[-12,12]$, $\Omega_{C F D} \subset \Omega_{a c}$, using an unstructured mesh of 421804 linear elements. The CFD and acoustic meshes coincide in the domain $\Omega_{C F D}$. The values for the constants in the stabilization parameters found in section 4.1 have been used for the simulations. Similarly to what has been done in that subsection, the following three situations have been considered to establish the influence of the various terms in the APE,

- Case C.1: No mean flow; the APE simplifies to the wave equation in mixed form. The sources terms are obtained from the CFD computation.

- Case C.2: The APE using the mean flow and source terms from the CFD computation but neglecting the reaction term. Namely, only non-uniform convection effects are considered.

- Case C.3: The full APE taking into account non-uniform convection and reaction terms.

In Fig. 9 we show the acoustic pressure contour levels at the far field for each case. As observed from Fig. 9a, a clear dipole radiation pattern emerges with waves propagating perpendicular to the incident inlet velocity, in Case C.1. However, when convection is taken into account (Case C.2) the wavefronts propagate upstream (see Fig. 9b) as expected [17], the effect being more pronounced if one includes reaction (Case C.3, Fig. 9c).

The differences in phase and amplitude among Cases C.1-C.3 can be also very clearly appreciated if we plot one-dimensional cuts of the domain in the $x, y$ and diagonal $x=y$ directions (see Fig. 10a, b and c). Again, this is also made apparent when plotting the time evolution of the acoustic pressure at three arbitrary points located at $\boldsymbol{x}_{1}=(-0.2,5.8)$, $\boldsymbol{x}_{2}=(3.1,0)$ and $\boldsymbol{x}_{3}=(9,0)$, see Figs. 11a, b and c.

\section{CONCLUSIONS}

The FEM solution of wave operators in mixed form requires using tailored finite elements that satisfy the discrete version of the continuous problem inf-sup condition. However, these finite elements are difficult to implement and though valid for the wave equation in mixed form, it has not been tested if they could work well for more complex operators, like the ones in the acoustic perturbation equations for low Mach numbers.

In this work, we have suggested to circumvent that problem by resorting to a stabilizing variational multiscale FEM formulation for the APE. The APE acoustic pressure and acoustic particle velocity get split into large scales, which can be resolved by the computational mesh and small scales whose influence onto the former has to be modelled. A residual-based model has been chosen for that purpose, the key for its good performance being the design of the matrix of stabilization parameters. The latter has been achieved by setting the small scales equations in the wavenumber domain and using appropriate inequality bounds for its norm as well as for the APE residual. The accomplishment of the proposed strategy has been checked by means of some numerical examples involving wave propagating in solenoidal non-uniform mean flows, involving convection and shear.

\section{ACKNOWLEDGMENTS}

This work is supported by EU-FET grant EUNISON 308874. The third author would like also to acknowledge the Generalitat de Catalunya (SUR/ECO) for the predoctoral FI Grant no. 2015 FI-B 00227. The fifth author acknowledges the support received from the Catalan Government through the ICREA Acadèmia Research Program. 


\section{APPENDIX A: BOUNDS ON THE ACOUSTIC PERTURBATION OPERATOR SPECTRAL RADIUS}

In this appendix bounds will be given for the spectral radii of the three terms in the r.h.s of (19). Without loss of generality, results will be derived for the two dimensional case, their extension to the three dimensional one being straightforward.

Taking into account the definitions of $\boldsymbol{A}_{i}$ in (5) and of the scaling matrix $\boldsymbol{M}$ in (13), the matrix in the first term of (19) reads

$$
\begin{aligned}
& \frac{1}{h^{2}} k_{i}^{0} k_{j}^{0} \boldsymbol{A}_{i}^{\top} \boldsymbol{M} \boldsymbol{A}_{j}=\frac{1}{h^{2}} \times \\
& \left(\begin{array}{ccc}
m_{\boldsymbol{u}}\left|\boldsymbol{k}^{0}\right|^{2}+\beta_{p}\left|\boldsymbol{k}^{0} \cdot \overline{\boldsymbol{u}}\right|^{2} & \alpha k_{1}^{0}\left(\boldsymbol{k}^{0} \cdot \overline{\boldsymbol{u}}\right) & \\
\alpha k_{1}^{0}\left(\boldsymbol{k}^{0} \cdot \overline{\boldsymbol{u}}\right) & m_{p}\left(k_{1}^{0}\right)^{2}+\beta_{\boldsymbol{u}}\left|\boldsymbol{k}^{0} \cdot \overline{\boldsymbol{u}}\right|^{2} & \alpha k_{2}^{0}\left(\boldsymbol{k}^{0} \cdot \overline{\boldsymbol{u}}\right) \\
\alpha k_{2}^{0}\left(\boldsymbol{k}^{0} \cdot \overline{\boldsymbol{u}}\right) & k_{1}^{0} k_{2}^{0} m_{p} & m_{p}\left(k_{2}^{0}\right)^{2}+\beta_{\boldsymbol{u}}\left|\boldsymbol{k}^{0} \cdot \overline{\boldsymbol{u}}\right|^{2}
\end{array}\right)
\end{aligned}
$$

with

$$
\alpha \equiv\left(m_{p} \mu_{p}+m_{\boldsymbol{u}} \mu_{\boldsymbol{u}}\right), \quad \beta_{p} \equiv m_{p} \mu_{p}^{2}, \quad \beta_{\boldsymbol{u}} \equiv m_{\boldsymbol{u}} \mu_{\boldsymbol{u}}^{2} .
$$

The spectrum of $k_{i}^{0} k_{j}^{0} \boldsymbol{A}_{i}^{\top} \boldsymbol{M} \boldsymbol{A}_{j}$ can be analytically worked out and is given by

$$
\operatorname{Spec}_{\boldsymbol{M}^{-1}}\left(k_{i}^{0} k_{j}^{0} \boldsymbol{A}_{i}^{\top} \boldsymbol{M} \boldsymbol{A}_{j}\right)=\left\{\left(\frac{\boldsymbol{k}^{0} \cdot \overline{\boldsymbol{u}}}{c_{0}}+\left|\boldsymbol{k}^{0}\right|\right)^{2},\left(\frac{\boldsymbol{k}^{0} \cdot \overline{\boldsymbol{u}}}{c_{0}}\right)^{2},\left(\frac{\boldsymbol{k}^{0} \cdot \overline{\boldsymbol{u}}}{c_{0}}-\left|\boldsymbol{k}^{0}\right|\right)^{2}\right\} .
$$

The spectral radius of $\left(\frac{1}{h^{2}} k_{l}^{0} k_{j}^{0} \boldsymbol{A}_{l}^{\top} \boldsymbol{M} \boldsymbol{A}_{j}\right)$ corresponds to the maximum eigenvalue

$$
\rho\left(\frac{1}{h^{2}} k_{l}^{0} k_{j}^{0} \boldsymbol{A}_{l}^{\top} \boldsymbol{M} \boldsymbol{A}_{j}\right)=\frac{1}{h^{2}}\left(\frac{\boldsymbol{k}^{0} \cdot \overline{\boldsymbol{u}}}{c_{0}}+\left|\boldsymbol{k}^{0}\right|\right)^{2} \equiv \frac{1}{h^{2}}\left(\frac{C_{2}|\overline{\boldsymbol{u}}|}{c_{0}}+C_{1}\right)^{2}=\frac{1}{h^{2}}\left(C_{1}+C_{2} M\right)^{2},
$$

where $M=|\overline{\boldsymbol{u}}| / c_{0}$ stands for the flow local Mach number (do not confuse the scalar Mach number $M$ with the weighthing matrix $\boldsymbol{M}$ ). Since $\boldsymbol{k}^{0}$ is an unknown dimensionless number, we have taken $\boldsymbol{k}^{0} \cdot \overline{\boldsymbol{u}}=\left|\boldsymbol{k}^{0}\right||\overline{\boldsymbol{u}}| \cos \theta \equiv C_{2}|\overline{\boldsymbol{u}}|$ and $\left|\boldsymbol{k}^{0}\right| \equiv C_{1}$ in the r.h.s of (A.4), $C_{1}$ and $C_{2}$ being dimensionless constants that have to be determined from numerical experiments.

Next, considering the expressions for $\boldsymbol{A}_{i}$ and $\boldsymbol{S}$ in (5) and for $\boldsymbol{M}$ in (13), we get for the matrix in the second term of (19)

$$
\begin{aligned}
& 2 \frac{\mathrm{i}}{h} k_{l}^{0}\left[\boldsymbol{A}_{l} \boldsymbol{M S}\right]_{A}=\frac{\mathrm{i}}{h} \times \\
& 0 \\
& \left(\begin{array}{ccc}
0 & m_{\boldsymbol{u}} \mu_{\boldsymbol{u}} \boldsymbol{k}^{0} \cdot \partial_{1} \overline{\boldsymbol{u}} & m_{\boldsymbol{u}} \mu_{\boldsymbol{u}} \boldsymbol{k}^{0} \cdot \partial_{2} \overline{\boldsymbol{u}} \\
-m_{\boldsymbol{u}} \mu_{\boldsymbol{u}} \boldsymbol{k}^{0} \cdot \partial_{1} \overline{\boldsymbol{u}} & 0 & -\beta_{\boldsymbol{u}} \boldsymbol{k}^{0} \cdot \overline{\boldsymbol{u}}\left(\partial_{1} \bar{u}_{2}-\partial_{2} \bar{u}_{1}\right) \\
-m_{\boldsymbol{u}} \mu_{\boldsymbol{u}} \boldsymbol{k}^{0} \cdot \partial_{2} \overline{\boldsymbol{u}} & \beta_{\boldsymbol{u}} \boldsymbol{k}^{0} \cdot \overline{\boldsymbol{u}}\left(\partial_{1} \bar{u}_{2}-\partial_{2} \bar{u}_{1}\right) & 0
\end{array}\right) .
\end{aligned}
$$

The spectrum of this matrix is given by

$$
\operatorname{Spec}_{\boldsymbol{M}^{-1}}\left(2 \frac{\mathrm{i}}{h} k_{l}^{0}\left[\boldsymbol{A}_{l} \boldsymbol{M} \boldsymbol{S}\right]_{A}\right)=\left\{\frac{1}{h}\left[\left(c^{2}+b^{2}\right)+m_{\boldsymbol{u}} d^{2}\right]^{1 / 2}, 0,-\frac{1}{h}\left[\left(c^{2}+b^{2}\right)+m_{\boldsymbol{u}} d^{2}\right]^{1 / 2}\right\},
$$

with

$$
\begin{aligned}
& c^{2}+b^{2}=\frac{1}{c_{0}^{2}}\left[\left(\boldsymbol{k}^{0} \cdot \partial_{1} \overline{\boldsymbol{u}}\right)^{2}+\left(\boldsymbol{k}^{0} \cdot \partial_{2} \overline{\boldsymbol{u}}\right)^{2}\right] \leq \frac{1}{c_{0}^{2}}\left|\boldsymbol{k}^{0}\right|^{2}|\nabla \overline{\boldsymbol{u}}|_{F}^{2}, \\
& m_{\boldsymbol{u}}^{2} d^{2}=m_{\boldsymbol{u}}^{2} \beta_{\boldsymbol{u}}\left(\boldsymbol{k}^{0} \cdot \overline{\boldsymbol{u}}\right)^{2}\left(\partial_{1} \bar{u}_{2}-\partial_{2} \bar{u}_{1}\right)^{2} \leq \frac{1}{c_{0}^{4}}\left|\boldsymbol{k}^{0}\right|^{2}|\overline{\boldsymbol{u}}|^{2}\left(\partial_{1} \bar{u}_{2}-\partial_{2} \bar{u}_{1}\right)^{2} \leq \frac{1}{c_{0}^{2}}\left|\boldsymbol{k}^{0}\right|^{2} M^{2}|\nabla \overline{\boldsymbol{u}}|_{F}^{2},
\end{aligned}
$$


$|\boldsymbol{B}|_{F}$ denoting the Frobenius norm of an arbitrary matrix $\boldsymbol{B}$. It follows that

$$
c^{2}+b^{2}+m_{\boldsymbol{u}}^{2} d^{2} \leq \frac{1}{c_{0}^{2}}\left|\boldsymbol{k}^{0}\right|^{2}|\nabla \overline{\boldsymbol{u}}|_{F}^{2}\left(1+M^{2}\right) \leq \frac{2}{c_{0}^{2}}\left|\boldsymbol{k}^{0}\right|^{2}|\nabla \overline{\boldsymbol{u}}|_{F}^{2},
$$

where we have considered that we are dealing with low Mach number flows and consequently $M<<1$. The above results allow one to bound the spectral radius of $2 \frac{\mathrm{i}}{h} k_{l}^{0}\left[\boldsymbol{A}_{l} \boldsymbol{M} \boldsymbol{S}\right]_{A}$ by

$$
\rho\left(2 \frac{\mathrm{i}}{h} k_{l}^{0}\left[\boldsymbol{A}_{l} \boldsymbol{M} \boldsymbol{S}\right]_{A}\right) \leq \frac{1}{h} \frac{C_{3}}{c_{0}}|\nabla \overline{\boldsymbol{u}}|_{F},
$$

$C_{3}$ denoting a dimensionless real constant.

Finally, the matrix in the third term of (19) reads

$$
\boldsymbol{S}^{\top} \boldsymbol{M} \boldsymbol{S}=\left(\begin{array}{ccc}
0 & 0 & 0 \\
0 & \beta_{\boldsymbol{u}}\left|\partial_{1} \overline{\boldsymbol{u}}\right|^{2} & \beta_{\boldsymbol{u}} \partial_{1} \overline{\boldsymbol{u}} \cdot \partial_{2} \overline{\boldsymbol{u}} \\
0 & \beta_{\boldsymbol{u}} \partial_{1} \overline{\boldsymbol{u}} \cdot \partial_{2} \overline{\boldsymbol{u}} & \beta_{\boldsymbol{u}}\left|\partial_{2} \overline{\boldsymbol{u}}\right|^{2}
\end{array}\right)
$$

with spectrum

$$
\operatorname{Spec}_{\boldsymbol{M}^{-1}}\left(\boldsymbol{S}^{\top} \boldsymbol{M S}\right)=\left\{\frac { m _ { \boldsymbol { u } } } { 2 } \left[(e+f)+\sqrt{(e-f)^{2}+4 g^{2}}, 0, \frac{m_{\boldsymbol{u}}}{2}\left[(e+f)-\sqrt{(e-f)^{2}+4 g^{2}}\right\},\right.\right.
$$

where

$$
\begin{aligned}
& (e+f)=\beta_{\boldsymbol{u}}\left[\left(\partial_{1} \overline{\boldsymbol{u}}\right)^{2}+\left(\partial_{2} \overline{\boldsymbol{u}}\right)^{2}\right] \leq \beta_{\boldsymbol{u}}|\nabla \overline{\boldsymbol{u}}|_{F}^{2}, \\
& (e-f)^{2}=\beta_{\boldsymbol{u}}^{2}\left[\left|\partial_{1} \overline{\boldsymbol{u}}\right|^{2}-\left|\partial_{2} \overline{\boldsymbol{u}}\right|^{2}\right]^{2} \leq \beta_{\boldsymbol{u}}^{2}|\nabla \overline{\boldsymbol{u}}|_{F}^{4}, \\
& 4 g^{2}=4 \beta_{\boldsymbol{u}}^{2}\left(\partial_{1} \overline{\boldsymbol{u}} \cdot \partial_{2} \overline{\boldsymbol{u}}\right)^{2} \leq 4 \beta_{\boldsymbol{u}}^{2}\left|\partial_{1} \overline{\boldsymbol{u}}\right|^{2}\left|\partial_{2} \overline{\boldsymbol{u}}\right|^{2} \leq 4 \beta_{\boldsymbol{u}}^{2}|\nabla \overline{\boldsymbol{u}}|_{F}^{4} .
\end{aligned}
$$

Therefore, the spectral radius of $\boldsymbol{S}^{\top} \boldsymbol{M} \boldsymbol{S}$ can be bounded as

$$
\rho\left(\boldsymbol{S}^{\top} \boldsymbol{M} \boldsymbol{S}\right) \leq C_{4} m_{\boldsymbol{u}} \beta_{\boldsymbol{u}}|\nabla \overline{\boldsymbol{u}}|_{F}^{2}=\frac{C_{4}}{c_{0}^{2}}|\nabla \overline{\boldsymbol{u}}|_{F}^{2},
$$

with $C_{4}$ denoting a large enough dimensionless real constant to be found again from numerical experiments.

From (A.4), (A.9) and (A.13) we get

$$
\begin{aligned}
& \rho\left(\frac{1}{h^{2}} k_{l}^{0} k_{j}^{0} \boldsymbol{A}_{l}^{\top} \boldsymbol{M} \boldsymbol{A}_{j}\right)+\rho\left(2 \frac{\mathrm{i}}{h} k_{l}^{0}\left[\boldsymbol{A}_{l} \boldsymbol{M} \boldsymbol{S}\right]_{A}\right)+\rho\left(\boldsymbol{S}^{\top} \boldsymbol{M} \boldsymbol{S}\right) \\
& \leq \frac{1}{h^{2}}\left(C_{1}+C_{2} M\right)^{2}+\frac{1}{h} \frac{C_{3}}{c_{0}}|\nabla \overline{\boldsymbol{u}}|_{F}+\frac{C_{4}}{c_{0}^{2}}|\nabla \overline{\boldsymbol{u}}|_{F}^{2} .
\end{aligned}
$$

\section{REFERENCES}

1. Ewert R, Schröder W. Acoustic perturbation equations based on flow decomposition via source filtering. J. Comput. Phys. 2003; 188(2):365-398.

2. Lighthill MJ. On sound generated aerodynamically I. general theory. Proc. R. Soc. Lond. A 1952; 211(1107):564-587.

3. Powell A. Theory of vortex sound. J. Acoust. Soc. Am. 1964; 36(1):177-195.

4. Howe M. The generation of sound by aerodynamic sources in an inhomogeneous steady flow. $J$. Fluid Mech. 1975; 67(03):597-610.

5. Möhring W. On vortex sound at low Mach number. J. Fluid Mech. 1978; 85(04):685-691.

6. Curle N. The influence of solid boundaries upon aerodynamic sound. Proc. R. Soc. Lond. A 1955; 231(1187):505-514. 
7. Williams JF, Hawkings DL. Sound generation by turbulence and surfaces in arbitrary motion. Phil. Trans. Roy. Soc. A 1969; 264(1151):321-342.

8. Bailly C, Bogey C, Gloerfelt X. Some useful hybrid approaches for predicting aerodynamic noise. C. R. Mec. 2005; 333(9):666-675.

9. Lilley G. On the noise radiated from a turbulent high speed jet, in Computational Aeroacoustics, J.C. Hardin and M.Y. Hussaini (Eds.). Springer Verlag, 1993.

10. Goldstein M. A generalized acoustic analogy. J. Fluid Mech. 2003; 488:315-333.

11. Bailly C, Bogey C. Contributions of computational aeroacoustics to jet noise research and prediction. Int. J. Comput. Fluid Dyn. 2004; 18(6):481-491.

12. Slimon SA, Soteriou MC, Davis DW. Development of computational aeroacoustics equations for subsonic flows using a mach number expansion approach. J. Comput. Phys. 2000; 159(2):377-406.

13. Oberai AA, Roknaldin F, R Hughes TJ. Computation of trailing-edge noise due to turbulent flow over an airfoil. AIAA J. 2002; 40(11):2206-2216.

14. Guasch O, Codina R. Computational aeroacoustics of viscous low speed flows using subgrid scale finite element methods. J. Comput. Acoust. 2009; 17(3):309-330.

15. Kaltenbacher M, Escobar M, Becker S, Ali I. Numerical simulation of flow-induced noise using LES/SAS and lighthill's acoustic analogy. Int. J. Numer. Meth. Fluids 2010; 63(9):1103-1122.

16. Baiges J, Codina R. A variational multiscale method with subscales on the element boundaries for the Helmholtz equation. Int. J. Numer. Meth. Engrg. 2013; 93(6):664-684.

17. Guasch O, Codina R. An algebraic subgrid scale finite element method for the convected helmholtz equation in two dimensions with applications in aeroacoustics. Comput. Methods Appl. Mech. Engrg. 2007; 196(45-48):4672-4689.

18. Hueppe A, Kaltenbacher M. Spectral finite elements for computational aeroacoustics using acoustic perturbation equations. J. Comput. Acoust. 2012; 20(02):1240 005.

19. Harari I. A survey of finite element methods for time-harmonic acoustics. Comput. Methods Appl. Mech. Engrg. 2006; 195(13):1594-1607.

20. Bécache E, Joly P, Tsogka C. An analysis of new mixed finite elements for the approximation of wave propagation problems. SIAM J. Numer. Anal. 2000; 37(4):1053-1084.

21. Bécache E, Joly P, Tsogka C. Fictitious domains, mixed finite elements and perfectly matched layers for 2-D elastic wave propagation. J. Comput. Acoust. 2001; 9(3):1175-1201.

22. Bécache E, Joly P, Tsogka C. A new family of mixed finite elements for the linear elastodynamic problem. SIAM J. Numer. Anal. 2002; 39(6):2109-2132.

23. Hughes T. Multiscale phenomena: Green's function, the dirichlet-to-neumann formulation, subgrid scale models, bubbles and the origins of stabilized formulations. Comput. Methods Appl. Mech. Engrg. 1995; 127:387-401.

24. Hughes T, Feijóo G, Mazzei L, Quincy J. The variational multiscale method, a paradigm for computational mechanics. Comput. Methods Appl. Mech. Engrg. 1998; 166:3-24.

25. Codina R. Stabilized finite element approximation of transient incompressible flows using orthogonal subscales. Comput. Methods Appl. Mech. Engrg. 2002; 191:4295-4321.

26. Codina R. Finite element approximation of the hyperbolic wave equation in mixed form. Comput. Methods Appl. Mech. Engrg. 2008; 197(13-16):1305-1322.

27. Badia S, Codina R, Espinoza H. Stability, convergence and accuracy of stabilized finite elements methods for the wave equation in mixed form. SIAM J. Numer. Anal. 2014; 52:1729-1752.

28. Langtangen HP, Pedersen G. Computational models for weakly dispersive nonlinear water waves. Comput. Methods Appl. Mech. Engrg. 1998; 160 (3-4):337-358.

29. Walkley M, Brezins,M. A finite element method for the two-dimensional extended Boussinesq equations. Int. J. Numer. Meth. Fluids 2002; 39(10):865-885.

30. Hauke G. A symmetric formulation for computing transient shallow water flows. Comput. Methods Appl. Mech. Engrg. 1998; 163 (1-4):111-122.

31. Codina R, González-Ondina J, Díaz-Hernández G, Principe J. Finite element approximation of the modified boussinesq equations using a stabilized formulation. Int. J. Numer. Meth. Fluids 2008; $\mathbf{5 7}(\mathbf{9}): 1249-1268$.

32. Guasch O, Arnela M, Codina R, Espinoza H. A stabilized finite element method for the mixed wave equation in an ALE framework with application to diphthong production. Acta Acust. 2016; 102(1):94-106.

33. Rao, PP, Morris, P. A stabilized finite element method for the mixed wave equation in an ALE framework with application to diphthong production. AIA A journal 2006; 44(7):1643-1652.

34. Iob, A, Arina, R, Schipani, C. Frequency-domain linearized Euler model for turbomachinery noise radiation through engine exhaust. AIAA journal 2010; 48(4):848-858.

35. Codina R, Principe J, Guasch O, Badia S. Time dependent subscsales in the stabilized finite element approximation of incompressible flow problems. Comput. Methods Appl. Mech. Engrg. 2007; 196(21-24):2413-2430.

36. Codina R, Principe J. Dynamic subscales in the finite element approximation of thermally coupled incompressible flows. Int. J. Numer. Meth. Fluids 2007; 54:707-730.

37. Ávila M, Codina R, Principe J. Large eddy simulation of low mach number flows using a dynamical and nonlinear finite element subgrid scale model. Comput. Fluids 2008; 57(9):1249-1268.

38. Takemoto H, Mokhtari P, Kitamura T. Acoustic analysis of the vocal tract during vowel production by finite-difference time-domain method. J. Acoust. Soc. Am. 2010; 128(6):3724-3738.

39. Codina R, Zienkiewicz O. CBS versus GLS stabilization of the incompressible Navier-Stokes equations and the role of the time step as stabilization parameter. Commun. Numer. Meth. Engrg. 2002; 18:99-112. 
40. Howe MS. Acoustics of fluid-structure interactions. Cambridge university press, 1998.

41. Drazin P. Introduction to Hydrodynamic Stability. Cambridge Texts in Applied Mathematics, Cambridge University Press, 2002. 